\title{
A Study of the Elliptic Restricted Three-Body Problem with Triaxial and Radiating Primaries Surrounded by a Belt
}

\author{
Jagadish Singh $^{1}$, Ndaman Isah ${ }^{2}$ \\ ${ }^{1}$ Department of Mathematics, Faculty of Physical Sciences, Ahmadu Bello University, Zaria, Kaduna State, Nigeria \\ ${ }^{2}$ Department of Mathematics, Faculty of Sciences, Niger State College of Education, Minna, Niger State, Nigeria
}

\section{Email address:}

jgds2004@yahoo.com (J. Singh), ndamaisah720@gmail.com (N. Isah)

\section{To cite this article:}

Jagadish Singh, Ndaman Isah. A Study of the Elliptic Restricted Three-Body Problem with Triaxial and Radiating Primaries Surrounded by a Belt. International Journal of Astrophysics and Space Science. Vol. 9, No. 1, 2021, pp. 1-16. doi: 10.11648/j.ijass.20210901.11

Received: September 28, 2020; Accepted: November 30, 2020; Published: January 22, 2021

\begin{abstract}
This paper examines the effects of radiation pressure and triaxiality of two stars (primaries) surrounded by a belt (circumbinary disc) on the positions and stability of a third body of an infinitesimal mass in the framework of the Elliptic restricted three body problem (ER3BP). We have obtained analytical solutions to the triangular equilibrium points and their stability and have also investigated these solutions numerically and graphically using radiating binary system (Xi- Bootis and Kruger 60). It is observed that their positions and stability are affected by semi-major axis, eccentricity of the orbit, triaxiality, radiation pressure of the primaries and potential from the belt. The perturbed parameters show the destabilizing tendency by decreasing the range of stability. The triangular points are found to bestable for $0<\mu<\mu_{c}$ where $\mu_{c}$ is the critical mass parameter. The stability analysis for the binary system yielded a stable outcome when we consider the range of mass parameter $\mu$ in the region of the Routhonian critical mass ratio (0.03852) when the effect of circumbinary disc is dominant. We found triaxiality and radition factors inducing instability even within this range.
\end{abstract}

Keywords: ER3BP, Triaxiality, Radiation Pressure, Pontetial from the Belt, Stability, Binary System, Routhonian Mass Ratio, Oblateness, Eccentricity, Semi-Major Axis, CR3BP

\section{Introduction}

The classical restricted three body problem assumes that the primary bodies are spherical in shape, whereas in the actual situation they are not. Most celestial bodies are oblate spheroids or triaxial rigid bodies. For Example, planets (Earth, Saturn and Jupiter) as well as stars (Archid, Luyten, Kruger60 and Xi-Bootis) are sufficiently oblate or triaxial rigid bodies and they play special and significant roles both in stellar and solar dynamics.In the elliptic restricted three body problem, the infinitesimal mass does not influence the motion of the primaries which move in elliptical orbits about their common centre of mass, but is influenced by them and lies in their gravitational field.

The elliptical restricted three-body problem has been studied by Nutan et al. [1], Szebehely [2] and Zimovschikov Thkai [3] afterwards certain specific characteristics of celestial bodies such as oblateness and triaxiality were taken into consideration (generalization). The reason being that such asphericity of celestial bodies causes perturbation, which is of interest to most astrometers and scientists. These have received the attention of [4-10]. They all studied the effect of perturbations on the orbit of the primaries with or without radiation pressure (s).

A similar problem studied by [11] considered the case where the three participating bodies are oblate spheroids.In [12] the stability of libration points when the smaller primary is a triaxial rigid body and the bigger one an oblate spheroids in the frame work of circular restricted three body problem (CRBBP) was determined. Also [13] studied the case where both primaries are oblate and radiating with gravitational pontential from a belt. In their paper,[14] established the equilibrium points and their stability when both primaries are oblate triaxial and sources of radiation as well in the elliptic restricted three body problems(ERT3B). Reference[15] included oblateness and triaxiality in their model, and they observed that there is a shift in the equilibrium points towards the line joining the primaries. 
Several studies under different assumptions have been conducted on the effect of circumbinary disc on the stability of equilibrium points. For example,[16] examined the analytic and numerical treatment of motion of adustgrain particle around triangular equilibrium points when the bigger primary is triaxial and the smaller one an oblate spheroid with a gravitational potential from the belt. They found that triangular points are stable for $0<\mu<\mu_{c}$ and unstable for $\mu_{c} \leq \mu \leq$ $\frac{1}{2}$, where $\mu$ subscriptc is the critical mass ratio. They also observed that the potential from the belt increases the range of stability. In their case, the triangular points no longer form equilateral triangles with the primaries but a scalene triangle due to perturbations. Similar results were obtained in [17] in terms of the region of stability, when the more massive primary is a source of radiation and the less massive primary is oblate with the potential from a belt.

[18] included P-R drag effect in their model when the first primaryis a triaxial body and the smaller one an oblate spheroid emitting radiation pressure, enclosed by a circumbinary disc. They concluded that the potential from the disc is a stabilizing force as it can change an unstable condition to stable one even when the mass parameter exceeds the critical mass value (that is, ).

In this paper, we study the effect of triaxiality and radiation pressure of the primaries on the third body of infinestimal mass in the framework of elliptical restricted three body problem (ER3BP) with potential from the belt. Since the orbits of most bodies are not spherical, analysing their motion in a circular restricted three body problem (CR3BP) would lead to exclusion of some parameters from the analysis, such as semi major axis and eccentricity.

Furthermore, the CR3BP is inadequate in describing the dynamics of a particle emitting radiation, because the gravitational force alone cannot be considered in studying the dynamics of a stellar system [19]. For Example, the gravity is not the major force present when a star collides with a gas and dust particles but the repelling forces of radiation pressures.

In the light of the above, we consider the motion of a test particle under the influences of two luminous and triaxial primaries moving in elliptic orbits enclosed by a circumbinary disc (belt). In this case we will use two binary system XiBootis and Kruger 60 for numerical explanation.

This paper is organized into the following sections: section 2 describes the equationsof motions; section 3 contains the solution to equilibrium points. In section 4 we obtain the stability, while we present numerical application in section 5 and section 6 is discussion and conclusion.

\section{Equation of Motion}

We present below the equations of motion of an infinesimal mass in the framework of ER3BP in which the primary and secondary bodies aretriaxial and radiating with potential from the belt. In a pulsating co-ordinate system with dimensionless variables $(\xi, \eta, \zeta)$ following [10],the equation of motion can be written as:

$$
\begin{aligned}
& \left.\begin{array}{c}
\xi^{\prime \prime}-2 \eta^{\prime}=\frac{\partial \Omega}{\partial \xi} \\
\eta^{\prime \prime}+2 \xi^{\prime}=\frac{\partial \Omega}{\partial \eta} \\
\zeta^{\prime \prime}=\frac{\partial \Omega}{\partial \zeta}
\end{array}\right\} \\
& \Omega=\left(1-e^{2}\right)^{-\frac{1}{2}}\left[\frac{1}{2}\left(\xi^{2}+\eta^{2}\right)+\frac{1}{n^{2}}\left\{\Omega_{1}+\Omega_{2}+\Omega_{3}\right\}\right]
\end{aligned}
$$

where

$$
\begin{aligned}
& \Omega_{1}=\frac{(1-\mu)}{r_{1}} q_{1}+\frac{(1-\mu)\left(2 \sigma_{1}-\sigma_{2}\right)}{2 r_{1}{ }^{3}} q_{1} \\
& \Omega_{2}=-\frac{3(1-\mu)\left(\sigma_{1}-\sigma_{2}\right)}{2 r_{1}{ }^{5}} q_{1} \eta^{2} \\
& \Omega_{3}=+\frac{\mu}{r_{2}} q_{2}+\frac{\mu\left(2 \sigma_{3}-\sigma_{4}\right)}{2 r_{2}{ }^{3}} q_{2}-\frac{3 \mu\left(\sigma_{3}-\sigma_{4}\right)}{2 r_{2}{ }^{5}} q_{2} \eta^{2}+\frac{M_{b}}{\left(r^{2}+T^{2}\right)^{\frac{1}{2}}} \\
& \left.\begin{array}{l}
r_{1}^{2}=(\xi+\mu)^{2}+\eta^{2}+\zeta^{2} \\
r_{2}^{2}=(\xi+\mu-1)^{2}+\eta^{2}+\zeta^{2}
\end{array}\right\} \\
& n^{2}=\frac{\left(1+e^{2}\right)^{\frac{1}{2}}}{a\left(1-e^{2}\right)}\left[1+\frac{2 M_{b} r_{c}}{\left(r_{c}^{2}+T^{2}\right)^{\frac{3}{2}}}+\frac{3}{2}\left(2 \sigma_{1}-\sigma_{2}\right)+\frac{3}{2}\left(2 \sigma_{3}-\sigma_{4}\right)\right]
\end{aligned}
$$

For simplicity

$$
n^{2}=\frac{1}{a}\left[1+\frac{3}{2} e^{2}+\frac{3}{2}\left(2 \sigma_{1}-\sigma_{2}\right)+\frac{3}{2}\left(2 \sigma_{3}-\sigma_{4}\right)+\frac{2 M_{b} r_{c}}{\left(r_{c}^{2}+T^{2}\right)^{\frac{3}{2}}}\right]
$$




$$
\left.\begin{array}{c}
\sigma_{1}=\frac{a^{2}-c^{2}}{5 R^{2}}, \sigma_{2}=\frac{a^{2}-c^{2}}{5 R^{2}}, \sigma_{3}=\frac{a^{\prime 2}-c^{\prime 2}}{5 R^{2}}, \sigma_{4}=\frac{a^{\prime 2}-c^{\prime 2}}{5 R^{2}}, \\
\mu=\frac{m_{2}}{m_{1}+m_{2}} \leq \frac{1}{2}, m_{1} \geq m_{2}, \sigma_{i} \ll 1,(i=1,2,3,4)
\end{array}\right\}
$$

where $\mu$ is the mass parameter, $\mathrm{n}$ is the mean motion of the primaries, $r_{1}$ and $r_{2}$ represent distances of the third body from the primaries, $\sigma_{1}$ and $\sigma_{2}$ denote the triaxiality of the bigger primary, while $\sigma_{3}$ and $\sigma_{4}$ denote the triaxiality of the smaller primary.

The lengths of the axis are denoted by a, b, c for the bigger primary and a', b', c' for the smaller primary, $r_{i},(i=1,2)$ are the distances of the infinitesimal mass from the bigger and smaller primaries respectively, while $q_{1}$ is the radiation factor of the bigger primary, $q_{2}$ the radiation factor of the smaller primary, $a$ is the semi-major axis of the orbits of the primaries and $e$ the enccentricity. $M_{\mathrm{b}}<<1$ is the total mass of the belt, $r$ is the radial distance of the infinitesimal mass given by $r^{2}=x^{2}+y^{2}, T=A+B$ and $B$ are the parameters which determine the density profile of the belt [20-22].
The parameter $B$ controls the size of the core of the density profile and is known as the core parameter. $r_{c}$ is the radial distance of the infinitesimal body through the triangular points in the classical R3BP.

\section{Location of Equilibrium Points}

The equilibrium points are the stationary solutions and are obtained by substituting $\xi^{\prime}=\eta^{\prime}=\zeta^{\prime}=\xi^{\prime \prime}=\eta^{\prime \prime}=\zeta^{\prime \prime}=0$ in the equations of motion (1). Thus, they are solutions of equations:

$$
\begin{aligned}
& \Omega_{\xi}=\left(1-e^{2}\right)^{-\frac{1}{2}}\left[\xi-\frac{1}{n^{2}}\left\{a_{1}+a_{2}\right\}\right]=0 \\
& \Omega_{\eta}=\left(1-e^{2}\right)^{-\frac{1}{2}}\left[\eta\left\{1-\frac{1}{n^{2}}\left(b_{1}+b_{2}\right)\right\}\right]=0 \\
& \Omega_{\zeta}=\left(1-e^{2}\right)^{-\frac{1}{2}}\left[-\frac{\zeta}{n^{2}}\left(c_{1}+c_{2}\right)\right]=0
\end{aligned}
$$

where

$$
\begin{aligned}
& a_{1}=\frac{(1-\mu)(\xi+\mu)}{r_{1}{ }^{3}} q_{1}+\frac{3(1-\mu)(\xi+\mu)\left(2 \sigma_{1}-\sigma_{2}\right)}{2 r_{1}{ }^{5}} q_{1}-\frac{15(1-\mu)(\xi+\mu)\left(\sigma_{1}-\sigma_{2}\right)}{2 r_{1}{ }^{7}} q_{1} \eta^{2} \\
& a_{2}=\frac{\mu(\xi+\mu-1)}{r_{2}{ }^{3}} q_{2}+\frac{3 \mu(\xi+\mu-1)\left(2 \sigma_{3}-\sigma_{4}\right)}{2 r_{2}{ }^{5}} q_{2}-\frac{15 \mu(\xi+\mu-1)\left(\sigma_{3}-\sigma_{4}\right)}{2 r_{2}{ }^{7}} q_{2} \eta^{2}-\frac{M_{b} \xi}{\left(r^{2}+T^{2}\right)^{\frac{3}{2}}} \\
& b_{1}=\frac{(1-\mu)}{r_{1}{ }^{3}} q_{1}+\frac{3(1-\mu)\left(2 \sigma_{1}-\sigma_{2}\right)}{2 r_{1}{ }^{5}} q_{1}-\frac{15(1-\mu)\left(\sigma_{1}-\sigma_{2}\right)}{2 r_{1}{ }^{7}} q_{1} \eta^{2}+\frac{3(1-\mu)\left(\sigma_{1}-\sigma_{2}\right)}{r_{1}{ }^{5}} q_{1} \\
& b_{2}=\frac{\mu}{r_{2}{ }^{3}} q_{2}+\frac{3 \mu\left(2 \sigma_{3}-\sigma_{4}\right)}{2 r_{2}{ }^{5}} q_{2}-\frac{15 \mu\left(\sigma_{3}-\sigma_{4}\right)}{2 r_{2}{ }^{7}} q_{2} \eta^{2}+\frac{3 \mu\left(\sigma_{3}-\sigma_{4}\right)}{r_{2}{ }^{5}} q_{2}+\frac{M_{b} \eta}{\left(r^{2}+T^{2}\right)^{\frac{3}{2}}} \\
& c_{1}=\frac{(1-\mu)}{r_{1}{ }^{3}} q_{1}+\frac{3(1-\mu)\left(2 \sigma_{1}-\sigma_{2}\right)}{2 r_{1}{ }^{5}} q_{1}-\frac{15(1-\mu)\left(\sigma_{1}-\sigma_{2}\right)}{2 r_{1}{ }^{7}} q_{1} \eta^{2} \\
& c_{2}=\frac{\mu}{r_{2}{ }^{3}} q_{2}+\frac{3 \mu\left(2 \sigma_{3}-\sigma_{4}\right)}{2 r_{2}{ }^{5}} q_{2}-\frac{15 \mu\left(\sigma_{3}-\sigma_{4}\right)}{2 r_{2}{ }^{7}} q_{2} \eta^{2}
\end{aligned}
$$

When triaxiality and the potential from the belt are absent, the first and the second of (6) can be written as:

$$
\begin{aligned}
& n^{2} \xi-\frac{(1-\mu)(\xi+\mu)}{r_{1}{ }^{3}} q_{1}-\frac{\mu(\xi+\mu-1)}{r_{2}{ }^{3}} q_{2}=0 \\
& n^{2}-\frac{(1-\mu)}{r_{1}{ }^{3}} q_{1}-\frac{\mu}{r_{2}{ }^{3}} q_{2}=0
\end{aligned}
$$

Using (7) and $\mu \neq 0$ we have

$$
r_{1}=\frac{q_{1}^{\frac{1}{3}}}{n^{\frac{2}{3}}}, \quad \mathrm{r}_{2}=\frac{q_{2^{\frac{1}{3}}}}{n^{\frac{2}{3}}}
$$

If we consider the effects of triaxiality of both primaries and the belt, the values of $r_{i}$ in (8) will change slightly by $\epsilon_{i(i=1,2)}$ where $\epsilon_{i}<<1$ and depends on triaxiality of both primaries and the potential from the belt so that:

$$
r_{1}=\frac{q_{1}^{\frac{1}{3}}}{n^{\frac{2}{3}}}+\varepsilon_{1}, \quad \mathrm{r}_{2}=\frac{q_{2}^{\frac{1}{3}}}{n^{\frac{2}{3}}}+\epsilon_{2}
$$

Using the values of $r_{1}$ and $r_{2}$ from (9) in (3) and neglecting the second and higher terms in $\epsilon_{i(i=1,2)}$ and their products with $e, \sigma_{i(i=1,2,3,4)}$ and $M_{b}$ we obtain:

$$
\begin{aligned}
\xi & =\frac{1}{2}-\mu+\frac{a^{\frac{2}{3}}\left(q_{1}^{\frac{2}{3}}-q_{2}{ }^{\frac{2}{3}}-q_{1} 1^{\frac{2}{3}} e^{2}+q_{2} 2^{\frac{2}{3}} e^{2}\right)}{2} \\
& +a^{\frac{1}{3}}\left(q_{1}^{\frac{1}{3}} \varepsilon_{1}-q_{2}{ }^{\frac{1}{3}} \varepsilon_{2}\right) \\
\eta^{2} & =\frac{\left(a q_{1}\right)^{\frac{2}{3}}\left(1-e^{2}\right)}{2}+\frac{\left(a q_{2}\right)^{\frac{2}{3}}\left(1-e^{2}\right)}{2} \\
& +a^{\frac{1}{3}}\left(q_{1}{ }^{\frac{1}{3}} \epsilon_{1}-q_{2}{ }^{\frac{1}{3}} \epsilon_{2}\right)-\frac{1}{4}
\end{aligned}
$$


Substituting the value of $\mathrm{n}^{2}$ from (4), $r_{i}(i=1,2)$ from (3) and $(\xi, \eta)$ from (10) into (6) and neglecting the second and higher order terms we have:

$$
\left.\begin{array}{l}
\varepsilon_{1}=A_{11}+A_{12}+A_{13} \\
\varepsilon_{2}=B_{11}+B_{12}+B_{13}+B_{14}
\end{array}\right\}
$$

where

$$
\begin{aligned}
A_{11}= & -\frac{\left(2 \sigma_{1}-\sigma_{2}\right)\left(a q_{1}\right)^{\frac{1}{3}}}{2}+\frac{\left(2 \sigma_{1}-\sigma_{2}\right)\left(a q_{1}\right)^{-\frac{1}{3}}}{2} \\
& -\frac{3\left(\sigma_{1}-\sigma_{2}\right)\left(a q_{2}\right)^{-\frac{1}{3}}}{4}-\frac{3\left(\sigma_{1}-\sigma_{2}\right)\left(a q_{2}\right)^{\frac{2}{3}}\left(a q_{1}\right)^{-\frac{1}{3}}}{4}-\frac{5\left(\sigma_{1}-\sigma_{2}\right)\left(a q_{2}\right)^{\frac{2}{3}}\left(a q_{1}\right)^{-1}}{4} \\
A_{12}= & \frac{5\left(\sigma_{1}-\sigma_{2}\right)\left(a q_{1}\right)^{-1}}{8}+\frac{5\left(\sigma_{1}-\sigma_{2}\right)\left(a q_{2}\right)^{\frac{4}{3}}\left(a q_{1}\right)^{-1}}{8}+\frac{\left(\sigma_{1}-\sigma_{2}\right)\left(a q_{1}\right)^{\frac{1}{3}}}{8}-\frac{\left(2 \sigma_{3}-\sigma_{4}\right)\left(a q_{1}\right)^{\frac{2}{3}}}{2} \\
A_{13}= & \frac{\mu\left(\sigma_{3}-\sigma_{4}\right)(\mathrm{aq})^{\frac{1}{3}}\left(a q_{2}\right)^{-\frac{2}{3}}}{2(1-\mu)}-\frac{\mu\left(\sigma_{3}-\sigma_{4}\right)\left(a q_{2}\right)^{-\frac{2}{3}}}{2(1-\mu)}+\frac{\mu\left(\sigma_{3}-\sigma_{4}\right)\left(a q_{1}\right)^{\frac{1}{3}}}{2(1-\mu)}-\frac{M_{b}\left(2 r_{c}-1\right)}{3\left(r_{c}{ }^{2}+T^{2}\right)^{\frac{3}{2}}} \\
B_{11}= & \frac{\left(2 \sigma_{1}-\sigma_{2}\right)\left(a q_{1}\right)^{\frac{1}{3}}}{2}-\frac{\left(2 \sigma_{3}-\sigma_{4}\right)\left(a q_{2}\right)^{\frac{1}{3}}}{2}+\frac{\left(\sigma_{3}-\sigma_{4}\right)\left(a q_{2}\right)^{-\frac{1}{3}}}{2}-\frac{5\left(\sigma_{3}-\sigma_{4}\right)\left(a q_{1}\right)^{\frac{2}{3}}\left(a q_{2}\right)^{-1}}{4} \\
B_{12}= & \frac{3\left(\sigma_{3}-\sigma_{4}\right)\left(a q_{2}\right)^{-\frac{1}{3}}}{16}+\frac{5\left(\sigma_{3}-\sigma_{4}\right)\left(a q_{2}\right)^{-1}}{8}+\frac{5\left(\sigma_{3}-\sigma_{4}\right)\left(a q_{1}\right)^{\frac{4}{3}}\left(a q_{2}\right)^{-1}}{8}-\frac{5\left(\sigma_{3}-\sigma_{4}\right)\left(a q_{2}\right)}{16} \\
B_{13}= & \frac{\left(\sigma_{1}-\sigma_{2}\right)\left(a q_{2}\right)^{\frac{1}{3}}\left(a q_{1}\right)^{-\frac{2}{3}}}{2 \mu}-\frac{\left(\sigma_{1}-\sigma_{2}\right)\left(a q_{2}\right)^{\frac{1}{3}}\left(a q_{1}\right)^{-\frac{2}{3}}}{2} \\
B_{14}= & \frac{\left(\sigma_{1}-\sigma_{2}\right)\left(a q_{2}\right)^{\frac{1}{3}}}{2 \mu}-\frac{\left(\sigma_{1}-\sigma_{2}\right)\left(a q_{2}\right)^{\frac{1}{3}}}{2}-\frac{\left(\sigma_{1}-\sigma_{2}\right)\left(a q_{2}\right)\left(a q_{1}\right)^{-\frac{2}{3}}}{2 \mu} \\
& +\frac{\left(\sigma_{1}-\sigma_{2}\right)\left(a q_{2}\right)\left(a q_{1}\right)^{-\frac{2}{3}}}{2}-\frac{3\left(\sigma_{3}-\sigma_{4}\right)\left(a q_{1}\right)^{\frac{2}{3}}\left(a q_{2}\right)^{-\frac{1}{3}}}{4}-\frac{5\left(\sigma_{3}-\sigma_{4}\right)\left(a q_{2}\right)^{\frac{2}{3}}}{8} \\
B_{15}= & \frac{\left(\sigma_{3}-\sigma_{4}\right)\left(a q_{2}\right)^{\frac{1}{3}}}{8}-\frac{\left(\sigma_{3}-\sigma_{4}\right)}{16}+\frac{5\left(\sigma_{3}-\sigma_{4}\right)\left(a q_{1}\right)^{\frac{2}{3}}\left(a q_{2}\right)^{\frac{1}{3}}}{16}-\frac{M_{b}\left(2 r_{c}-1\right)}{3\left(r_{c}^{2}+T^{2}\right)^{\frac{3}{2}}}
\end{aligned}
$$

Making use of (11) in (10) we obtain:

$$
\begin{gathered}
\xi=C_{00}+\left(C_{11}+C_{12}\right) \sigma_{1}+\left(C_{21}+C_{22}\right) \sigma_{2}+\left(C_{31}+C_{32}\right) \sigma_{3}+\left(C_{41}+C_{42}\right) \sigma_{4} \\
\eta= \pm\left[D_{00}+D_{A}+D_{B}+D_{5}\right]^{\frac{1}{2}} \\
C_{00}=\frac{1}{2}-\mu+\frac{\left(a q_{1}\right)^{\frac{2}{3}}}{2}-\frac{\left(a q_{2}\right)^{\frac{2}{3}}}{2}-\left[\frac{\left(a q_{1}\right)^{\frac{2}{3}}}{2}-\frac{\left(a q_{2}\right)^{\frac{2}{3}}}{2}\right] e^{2} \\
C_{11}=\frac{-7\left(a q_{1}\right)^{\frac{2}{3}}}{8}+\frac{1}{4}+\frac{3\left(a q_{2}\right)^{\frac{2}{3}}}{4}-\frac{3\left(a q_{2}\right)^{\frac{2}{3}}\left(a q_{1}\right)^{-\frac{2}{3}}}{4}+\frac{5\left(a q_{1}\right)^{-\frac{2}{3}}}{8} \\
C_{12}=\frac{\left(a q_{2}\right)^{\frac{4}{3}}\left(a q_{1}\right)^{-\frac{2}{3}}}{8}-\frac{\left(a q_{2}\right)^{\frac{2}{3}}\left(a q_{1}\right)^{-\frac{2}{3}}}{2 \mu}-\frac{\left(a q_{2}\right)^{\frac{2}{3}}}{2 \mu}+\frac{\left(a q_{2}\right)^{\frac{4}{3}}\left(a q_{1}\right)^{-\frac{2}{3}}}{2 \mu} \\
C_{21}=\frac{3\left(a q_{1}\right)^{\frac{2}{3}}}{8}+\frac{1}{4}-\frac{\left(a q_{2}\right)^{\frac{2}{3}}}{4}+\frac{3\left(a q_{2}\right)^{\frac{2}{3}}\left(a q_{1}\right)^{-\frac{2}{3}}}{4}-\frac{5\left(a q_{1}\right)^{-\frac{2}{3}}}{8} \\
C_{22}=-\frac{\left(a q_{2}\right)^{\frac{4}{3}}\left(a q_{1}\right)^{-\frac{2}{3}}}{8}+\frac{\left(a q_{2}\right)^{\frac{2}{3}}\left(a q_{1}\right)^{-\frac{2}{3}}}{2 \mu}+\frac{\left(a q_{2}\right)^{\frac{2}{3}}}{2 \mu}-\frac{\left(a q_{2}\right)^{\frac{4}{3}}\left(a q_{1}\right)^{-\frac{2}{3}}}{2 \mu}
\end{gathered}
$$




$$
\begin{aligned}
& C_{31}=\frac{-\left(a q_{1}\right)^{\frac{2}{3}}}{4}+\frac{\mu\left(a q_{1}\right)^{\frac{2}{3}}\left(a q_{2}\right)^{-\frac{2}{3}}}{2(1-\mu)}-\frac{\mu\left(a q_{1}\right)^{\frac{1}{3}}\left(a q_{2}\right)^{-\frac{2}{3}}}{2(1-\mu)}+\frac{\mu\left(a q_{1}\right)^{\frac{2}{3}}}{2(1-\mu)}+\frac{7\left(a q_{2}\right)^{\frac{2}{3}}}{8}+\frac{5\left(a q_{2}\right)^{-\frac{2}{3}}\left(a q_{1}\right)^{\frac{2}{3}}}{4} \\
& C_{32}=-\frac{19}{16}-\frac{5\left(a q_{2}\right)^{-\frac{2}{3}}}{8}-\frac{5\left(a q_{1}\right)^{\frac{4}{3}}\left(a q_{2}\right)^{-\frac{2}{3}}}{8}+\frac{5\left(a q_{2}\right)^{\frac{4}{3}}}{16}+\frac{5\left(a q_{2}\right)}{8}+\frac{5\left(a q_{2}\right)^{\frac{1}{3}}}{16}-\frac{5\left(a q_{1}\right)\left(a q_{2}\right)^{\frac{1}{3}}}{16} \\
& C_{41}=\frac{-\left(a q_{1}\right)^{\frac{2}{3}}}{4}-\frac{\mu\left(a q_{1}\right)^{\frac{2}{3}}\left(a q_{2}\right)^{-\frac{2}{3}}}{2(1-\mu)}+\frac{\mu\left(a q_{1}\right)^{\frac{1}{3}}\left(a q_{2}\right)^{-\frac{2}{3}}}{2(1-\mu)}-\frac{\mu\left(a q_{1}\right)^{\frac{2}{3}}}{2(1-\mu)}-\frac{3\left(a q_{2}\right)^{\frac{2}{3}}}{8}+\frac{11}{16} \\
& C_{42}=-\frac{5\left(a q_{2}\right)^{-\frac{2}{3}}\left(a q_{1}\right)^{\frac{2}{3}}}{4}+\frac{5\left(a q_{2}\right)^{-\frac{2}{3}}}{8}+\frac{5\left(a q_{1}\right)^{\frac{4}{3}}\left(a q_{2}\right)^{-\frac{2}{3}}}{8}-\frac{5\left(a q_{2}\right)^{\frac{4}{3}}}{16}-\frac{5\left(a q_{2}\right)}{8}-\frac{5\left(a q_{2}\right)^{\frac{1}{3}}}{16}+\frac{5\left(a q_{1}\right)\left(a q_{2}\right)^{\frac{1}{3}}}{16} \\
& D_{A}=\left(D_{11}+D_{12}\right) \sigma_{1}+\left(D_{21}+D_{22}\right) \sigma_{2} \\
& D_{B}=+\left(D_{31}+D_{32}\right) \sigma_{3}+\left(D_{41}+D_{42}\right) \sigma_{4} \\
& D_{00}=\frac{\left(a q_{1}\right)^{\frac{2}{3}}}{2}+\frac{\left(a q_{2}\right)^{\frac{2}{3}}}{2}-\left(\frac{\left(a q_{1}\right)^{\frac{2}{3}}}{2}+\frac{\left(a q_{2}\right)^{\frac{2}{3}}}{2}\right) e^{2}-\frac{1}{4} \\
& D_{11}=\frac{-7\left(a q_{1}\right)^{\frac{2}{3}}}{8}+\frac{1}{4}-\frac{9\left(a q_{2}\right)^{\frac{2}{3}}}{4}-\frac{5\left(a q_{2}\right)^{\frac{2}{3}}\left(a q_{1}\right)^{-\frac{2}{3}}}{4}+\frac{5\left(a q_{1}\right)^{-\frac{2}{3}}}{8}+\frac{5\left(a q_{2}\right)^{\frac{4}{3}}\left(a q_{1}\right)^{-\frac{2}{3}}}{8}+\frac{\left(a q_{2}\right)^{\frac{2}{3}}\left(a q_{1}\right)^{-\frac{2}{3}}}{2 \mu} \\
& D_{12}=-\frac{\left(a q_{2}\right)^{\frac{2}{3}}\left(a q_{1}\right)^{-\frac{2}{3}}}{2}+\frac{\left(a q_{2}\right)^{\frac{2}{3}}}{2 \mu}-\frac{\left(a q_{2}\right)^{\frac{4}{3}}\left(a q_{1}\right)^{-\frac{2}{3}}}{2 \mu}+\frac{\left(a q_{2}\right)^{\frac{4}{3}}\left(a q_{1}\right)^{-\frac{2}{3}}}{2} \\
& D_{21}=\frac{3\left(a q_{1}\right)^{\frac{2}{3}}}{8}+\frac{1}{4}+\frac{7\left(a q_{2}\right)^{\frac{2}{3}}}{4}+\frac{5\left(a q_{2}\right)^{\frac{2}{3}}\left(a q_{1}\right)^{-\frac{2}{3}}}{4}-\frac{5\left(a q_{1}\right)^{-\frac{2}{3}}}{8}-\frac{5\left(a q_{2}\right)^{\frac{4}{3}}\left(a q_{1}\right)^{-\frac{2}{3}}}{8}-\frac{\left(a q_{2}\right)^{\frac{2}{3}}\left(a q_{1}\right)^{-\frac{2}{3}}}{2 \mu} \\
& D_{22}=\frac{\left(a q_{2}\right)^{\frac{2}{3}}\left(a q_{1}\right)^{-\frac{2}{3}}}{2}-\frac{\left(a q_{2}\right)^{\frac{2}{3}}}{2 \mu}+\frac{\left(a q_{2}\right)^{\frac{4}{3}}\left(a q_{1}\right)^{-\frac{2}{3}}}{2 \mu}-\frac{\left(a q_{2}\right)^{\frac{4}{3}}\left(a q_{1}\right)^{-\frac{2}{3}}}{2} \\
& D_{31}=\frac{-7\left(a q_{1}\right)^{\frac{2}{3}}}{4}+\frac{\mu\left(a q_{1}\right)^{\frac{2}{3}}\left(a q_{2}\right)^{-\frac{2}{3}}}{2(1-\mu)}-\frac{\mu\left(a q_{1}\right)^{\frac{1}{3}}\left(a q_{2}\right)^{-\frac{2}{3}}}{2(1-\mu)}+\frac{\mu\left(\mathrm{aq}_{1}\right)^{\frac{2}{3}}}{2(1-\mu)}-\frac{7\left(\mathrm{aq}_{2}\right)^{\frac{2}{3}}}{8} \\
& D_{32}=-\frac{5\left(a q_{2}\right)^{-\frac{2}{3}}\left(a q_{1}\right)^{\frac{2}{3}}}{4}+\frac{19}{16}+\frac{5\left(a q_{2}\right)^{-\frac{2}{3}}}{8}+\frac{5\left(a q_{1}\right)^{\frac{4}{3}}\left(a q_{2}\right)^{-\frac{2}{3}}}{8}-\frac{5\left(a q_{2}\right)^{\frac{4}{3}}}{16}-\frac{5\left(a q_{2}\right)}{8}-\frac{5\left(a q_{2}\right)^{\frac{1}{3}}}{16}+\frac{5\left(a q_{1}\right)\left(a q_{2}\right)^{\frac{1}{3}}}{16} \\
& D_{41}=\frac{5\left(a q_{1}\right)^{\frac{2}{3}}}{4}-\frac{\mu\left(a q_{1}\right)^{\frac{2}{3}}\left(a q_{2}\right)^{-\frac{2}{3}}}{2(1-\mu)}+\frac{\mu\left(a q_{1}\right)^{\frac{1}{3}}\left(a q_{2}\right)^{-\frac{2}{3}}}{2(1-\mu)}-\frac{\mu\left(a q_{1}\right)^{\frac{2}{3}}}{2(1-\mu)}+\frac{3\left(a q_{2}\right)^{\frac{2}{3}}}{8} \\
& D_{42}=\frac{5\left(a q_{2}\right)^{-\frac{2}{3}}\left(a q_{1}\right)^{\frac{2}{3}}}{4}-\frac{11}{16}-\frac{5\left(a q_{2}\right)^{-\frac{2}{3}}}{8}-\frac{5\left(a q_{1}\right)^{\frac{4}{3}}\left(a q_{2}\right)^{-\frac{2}{3}}}{8}+\frac{5\left(a q_{2}\right)^{\frac{4}{3}}}{16}+\frac{5\left(a q_{2}\right)}{8}+\frac{5\left(a q_{2}\right)^{\frac{1}{3}}}{16}-\frac{5\left(a q_{2}\right)^{\frac{1}{2}}\left(a q_{1}\right)}{16} \\
& D_{50}=-\frac{4 M_{b}\left(2 r_{c-1}\right)}{3\left(r_{c}^{2}+T^{2}\right)^{\frac{3}{2}}}
\end{aligned}
$$

The Points above are the triangular points and are denoted by $L_{4,}$ and are known as Lagrangian triangular equilibrium points.

\section{Stability of Triangular Equilibrium Points $\mathrm{L}_{4,5}$}

We investigate the motion of a dust particle near the equilibrium point $\left(\xi_{0}, \eta_{0}\right)$ and consider small displacement $(\alpha, \beta)$ from this position so that $\xi=\xi_{0}+\alpha$ and $\eta=\eta_{0}+\beta$. When these values are substituted in equation (1) then the variational equation 
are obtained thus:

$$
\alpha^{\prime \prime}-2 \beta^{\prime}=\alpha \Omega_{\xi \xi}^{0}+\beta \Omega_{\xi \eta}^{0}, \beta^{\prime \prime}+2 \alpha^{\prime}=\alpha \Omega_{\xi \eta}^{0}+\beta \Omega_{\eta \eta}^{0}
$$

We consider only the linear terms in $\alpha$ and $\beta$. The second partial derivatives of the force function are denoted using subscripts. The superscripts $\mathrm{O}$ shows that the partial derivatives are taken at the equilibrium point $\left(\xi_{0}, \eta_{0}\right)$ Following the same linear stability analysis used in [9], the characteristic equation of the equilibrium point is

$$
\lambda^{4}-\left(\Omega_{\xi \xi}^{0}+\Omega_{\eta \eta}^{0}-4\right) \lambda^{2}+\left(\Omega_{\xi \xi}^{0}\right)\left(\Omega_{\xi \eta}^{0}\right)-\left(\Omega_{\xi \eta}^{0}\right)^{2}=0
$$

The second partial derivatesevaluated at equilibrium points Using equation (12) are:

$$
\left.\begin{array}{l}
\Omega^{0}{ }_{\xi \xi}=\left(1-e^{2}\right)^{-\frac{1}{2}}\left[E_{0}+E_{A}+E_{B}+E_{5}\right] \\
\Omega_{\eta \eta}{ }^{0}=\left(1-e^{2}\right)^{-\frac{1}{2}}\left[F_{A} \sigma_{1}+F_{B} \sigma_{2}+\left(F_{3}\right) \sigma_{3}+\left(F_{4}\right) \sigma_{4}+F_{5}\right] \\
\Omega_{\xi \eta}{ }^{0}=\left(1-e^{2}\right)^{-\frac{1}{2}} \eta\left[G_{0}+\left(G_{1}\right) \sigma_{1}+\left(G_{2}\right) \sigma_{2}+\left(G_{3}\right) \sigma_{3}+\left(G_{4}\right) \sigma_{4}+G_{5}\right]
\end{array}\right\}
$$

where

$$
\begin{aligned}
& E_{A}=\left(E_{11}+E_{12}+E_{13}\right) \sigma_{1}+\left(E_{21}+E_{22}\right) \sigma_{2} \\
& E_{B}=\left(E_{31}+E_{32}\right) \sigma_{3}+\left(E_{41}+E_{42}\right) \sigma_{4} \\
& F_{A}=F_{0}+F_{01}+F_{11}+F_{12} \\
& F_{B}=F_{21}+F_{22} \\
& E_{0}=\frac{3(1-\mu)}{4\left(a q_{1}\right)^{\frac{2}{3}}}+\frac{3(1-\mu)}{2}+\frac{3 \mu}{4\left(a q_{2}\right)^{\frac{2}{3}}}-\frac{3 \mu\left(a q_{1}\right)^{\frac{2}{3}}}{2\left(a q_{2}\right)^{\frac{2}{3}}}+\frac{3 \mu}{2}-\frac{3(1-\mu)\left(a q_{2}\right)^{\frac{2}{3}}}{2\left(a q_{1}\right)^{\frac{2}{3}}}+\left(\frac{3(1-\mu)}{4\left(a q_{1}\right)^{\frac{2}{3}}}+\frac{3 \mu}{4\left(a q_{2}\right)^{\frac{2}{3}}}\right) e^{2} \\
& E_{11}=\frac{-75}{16}+\frac{111 \mu}{16}+\frac{741}{32\left(a q_{1}\right)^{\frac{2}{3}}}-\frac{621 \mu}{32\left(a q_{1}\right)^{\frac{2}{3}}}+\frac{93\left(a q_{2}\right)^{\frac{2}{3}}}{16\left(a q_{1}\right)^{\frac{2}{3}}}-\frac{99 \mu\left(a q_{2}\right)^{\frac{2}{3}}}{16\left(a q_{1}\right)^{\frac{2}{3}}}+\frac{93\left(a q_{2}\right)^{\frac{2}{3}}}{16\left(a q_{1}\right)^{\frac{4}{3}}}-\frac{69 \mu\left(a q_{2}\right)^{\frac{2}{3}}}{16\left(a q_{1}\right)^{\frac{4}{3}}} \\
& E_{12}=-\frac{15(1-\mu)\left(a q_{2}\right)^{\frac{2}{3}}}{4\left(a q_{1}\right)^{2}}+\frac{15(1-\mu)}{16\left(a q_{1}\right)^{2}}+\frac{45(1-\mu)\left(a q_{2}\right)^{\frac{4}{3}}}{32\left(a q_{1}\right)^{2}} \\
& -\frac{9 \mu}{8\left(a q_{2}\right)^{\frac{2}{3}}}-\frac{33 \mu\left(a q_{1}\right)^{\frac{2}{3}}}{32\left(a q_{2}\right)^{\frac{2}{3}}}-\frac{153\left(a q_{2}\right)^{\frac{4}{3}}}{16\left(a q_{1}\right)^{\frac{4}{3}}} \frac{129\left(a q_{2}\right)^{\frac{4}{3}}}{16\left(a q_{1}\right)^{\frac{4}{3}}}+\frac{75(1-\mu)\left(a q_{2}\right)^{2}}{16\left(a q_{1}\right)^{2}} \\
& E_{13}=-\frac{3\left(a q_{2}\right)^{\frac{2}{3}}}{2 \mu\left(a q_{1}\right)^{\frac{4}{3}}}-\frac{3\left(a q_{2}\right)^{\frac{2}{3}}}{2 \mu\left(a q_{1}\right)^{\frac{2}{3}}}+\frac{3\left(a q_{2}\right)^{\frac{4}{3}}}{2 \mu\left(a q_{1}\right)^{\frac{4}{3}}}-\frac{15}{8\left(a q_{2}\right)^{\frac{2}{3}}\left(a q_{1}\right)^{\frac{2}{3}}}+\frac{15}{8\left(a q_{2}\right)^{\frac{2}{3}}}+\frac{15\left(a q_{1}\right)^{\frac{2}{3}}}{4\left(a q_{2}\right)^{\frac{2}{3}}} \\
& E_{21}=\frac{75}{16}-\frac{111 \mu}{16}-\frac{669}{32\left(a q_{1}\right)^{\frac{2}{3}}}+\frac{549 \mu}{32\left(a q_{1}\right)^{\frac{2}{3}}}-\frac{93\left(a q_{2}\right)^{\frac{2}{3}}}{16\left(a q_{1}\right)^{\frac{2}{3}}} \\
& +\frac{99 \mu\left(a q_{2}\right)^{\frac{2}{3}}}{16\left(a q_{1}\right)^{\frac{2}{3}}}-\frac{93\left(a q_{2}\right)^{\frac{2}{3}}}{16\left(a q_{1}\right)^{\frac{4}{3}}}+\frac{69 \mu\left(a q_{2}\right)^{\frac{2}{3}}}{16\left(a q_{1}\right)^{\frac{4}{3}}}+\frac{15(1-\mu)\left(a q_{2}\right)^{\frac{2}{3}}}{4\left(a q_{1}\right)^{2}}-\frac{15(1-\mu)}{16\left(a q_{1}\right)^{2}}-\frac{45(1-\mu)\left(a q_{2}\right)^{\frac{4}{3}}}{32\left(a q_{1}\right)^{2}} \\
& E_{22}=\frac{3 \mu}{8\left(a q_{2}\right)^{\frac{2}{3}}}+\frac{33 \mu\left(a q_{1}\right)^{\frac{2}{3}}}{8\left(a q_{2}\right)^{\frac{2}{3}}}+\frac{153\left(a q_{2}\right)^{\frac{4}{3}}}{16\left(a q_{1}\right)^{\frac{4}{3}}}-\frac{129 \mu\left(a q_{2}\right)^{\frac{4}{3}}}{16\left(a q_{1}\right)^{\frac{4}{3}}} \\
& -\frac{75(1-\mu)\left(a q_{2}\right)^{2}}{16\left(a q_{1}\right)^{2}}+\frac{3\left(a q_{2}\right)^{\frac{2}{3}}}{2 \mu\left(a q_{1}\right)^{\frac{4}{3}}}+\frac{3\left(a q_{2}\right)^{\frac{2}{3}}}{2 \mu\left(a q_{1}\right)^{\frac{2}{3}}}-\frac{3\left(a q_{2}\right)^{\frac{4}{3}}}{2 \mu\left(a q_{1}\right)^{\frac{4}{3}}}+\frac{15}{8\left(a q_{1}\right)^{\frac{2}{3}}\left(a q_{2}\right)^{\frac{2}{3}}}-\frac{15}{8\left(a q_{2}\right)^{\frac{2}{3}}}-\frac{15\left(a q_{1}\right)^{\frac{2}{3}}}{4\left(a q_{2}\right)^{\frac{2}{3}}}
\end{aligned}
$$




$$
\begin{aligned}
& E_{31}=\frac{9}{4}-\frac{129 \mu}{64}-\frac{33}{16\left(a q_{1}\right)^{\frac{2}{3}}}+\frac{63 \mu}{16\left(a q_{1}\right)^{\frac{2}{3}}}-\frac{3\left(a q_{2}\right)^{\frac{2}{3}}}{8\left(a q_{1}\right)^{\frac{2}{3}}}+\frac{33 \mu\left(a q_{2}\right)^{\frac{2}{3}}}{8\left(a q_{1}\right)^{\frac{2}{3}}}-\frac{81 \mu}{8\left(a q_{2}\right)^{\frac{2}{3}}} \\
& +\frac{177 \mu\left(a q_{1}\right)^{\frac{2}{3}}}{64\left(a q_{2}\right)^{\frac{2}{3}}}-\frac{225 \mu}{64\left(a q_{2}\right)^{\frac{4}{3}}}+\frac{315 \mu\left(a q_{1}\right)^{\frac{2}{3}}}{32\left(a q_{2}\right)^{\frac{4}{3}}}-\frac{45 \mu\left(a q_{1}\right)^{\frac{2}{3}}}{4\left(a q_{2}\right)^{2}}+\frac{45 \mu\left(a q_{1}\right)^{\frac{4}{3}}}{32\left(a q_{2}\right)^{2}}+\frac{15 \mu}{16\left(a q_{2}\right)^{2}} \\
& +\frac{15 \mu}{8\left(a q_{2}\right)^{\frac{2}{3}}\left(a q_{1}\right)}-\frac{3 \mu}{4\left(a q_{2}\right)^{\frac{2}{3}}\left(a q_{1}\right)^{\frac{1}{3}}}-\frac{15 \mu}{4\left(a q_{1}\right)}-\frac{3 \mu^{2}\left(a q_{1}\right)^{\frac{2}{3}}}{2(1-\mu)\left(a q_{2}\right)^{\frac{4}{3}}}+\frac{3 \mu^{2}\left(a q_{1}\right)^{\frac{1}{3}}}{2(1-\mu)\left(a q_{2}\right)^{\frac{4}{3}}} \\
& -\frac{3 \mu^{2}\left(a q_{1}\right)^{\frac{2}{3}}}{2(1-\mu)\left(a q_{2}\right)^{\frac{2}{3}}}+\frac{15}{4\left(a q_{2}\right)^{\frac{2}{3}}}-\frac{15}{8\left(a q_{1}\right)^{\frac{2}{3}}\left(a q_{2}\right)^{\frac{2}{3}}}-\frac{15\left(a q_{1}\right)^{\frac{2}{3}}}{8\left(a q_{2}\right)^{\frac{2}{3}}}+\frac{15(1-\mu)\left(a q_{2}\right)^{\frac{4}{3}}}{16\left(a q_{1}\right)^{\frac{2}{3}}} \\
& E_{32}=\frac{15(1-\mu)\left(a q_{2}\right)}{8\left(a q_{1}\right)^{\frac{2}{3}}}+\frac{15(1-\mu)\left(a q_{2}\right)^{\frac{1}{3}}}{16\left(a q_{1}\right)^{\frac{2}{3}}}-\frac{15\left(a q_{2}\right)^{\frac{2}{3}}}{16}+\frac{45 \mu\left(a q_{2}\right)^{\frac{2}{3}}}{32}-\frac{105 \mu\left(a q_{1}\right)^{\frac{4}{3}}}{16\left(a q_{2}\right)^{\frac{4}{3}}} \\
& +\frac{15 \mu\left(a q_{2}\right)^{\frac{1}{3}}}{16}+\frac{15 \mu}{16\left(a q_{2}\right)^{\frac{1}{3}}}-\frac{45 \mu\left(a q_{1}\right)^{\frac{2}{3}}}{16}+\frac{75 \mu}{64\left(a q_{2}\right)}+\frac{75 \mu\left(a q_{1}\right)^{2}}{16\left(a q_{2}\right)^{2}}-\frac{75 \mu\left(a q_{1}\right)^{\frac{2}{3}}}{16\left(a q_{2}\right)^{\frac{1}{3}}}-\frac{75 \mu\left(a q_{1}\right)^{\frac{2}{3}}}{32\left(a q_{2}\right)}+\frac{75 \mu\left(a q_{1}\right)^{\frac{4}{3}}}{32\left(a q_{2}\right)^{\frac{2}{3}}} \\
& E_{41}=-\frac{9}{4}+\frac{129 \mu}{64}+\frac{21}{16\left(a q_{1}\right)^{\frac{2}{3}}}-\frac{51 \mu}{16\left(a q_{1}\right)^{\frac{2}{3}}}+\frac{3\left(a q_{2}\right)^{\frac{2}{3}}}{8\left(a q_{1}\right)^{\frac{2}{3}}}-\frac{33 \mu\left(a q_{2}\right)^{\frac{2}{3}}}{8\left(a q_{1}\right)^{\frac{2}{3}}} \\
& +\frac{99 \mu}{8\left(a q_{2}\right)^{\frac{2}{3}}}-\frac{177 \mu\left(a q_{1}\right)^{\frac{2}{3}}}{64\left(a q_{2}\right)^{\frac{2}{3}}}+\frac{225 \mu}{64\left(a q_{2}\right)^{\frac{4}{3}}}-\frac{315 \mu\left(a q_{1}\right)^{\frac{2}{3}}}{32\left(a q_{2}\right)^{\frac{4}{3}}}+\frac{45 \mu\left(a q_{1}\right)^{\frac{2}{3}}}{4\left(a q_{2}\right)^{2}}-\frac{45 \mu\left(a q_{1}\right)^{\frac{4}{3}}}{32\left(a q_{2}\right)^{2}} \\
& -\frac{15 \mu}{16\left(a q_{2}\right)^{2}}-\frac{15 \mu}{8\left(a q_{2}\right)^{\frac{2}{3}}\left(a q_{1}\right)}-\frac{3 \mu}{4\left(a q_{2}\right)^{\frac{2}{3}}\left(a q_{1}\right)^{\frac{1}{3}}}+\frac{15 \mu}{4\left(a q_{1}\right)}+\frac{3 \mu^{2}\left(a q_{1}\right)^{\frac{2}{3}}}{2(1-\mu)\left(a q_{2}\right)^{\frac{4}{3}}}-\frac{3 \mu^{2}\left(a q_{1}\right)^{\frac{1}{3}}}{2(1-\mu)\left(a q_{2}\right)^{\frac{4}{3}}} \\
& +\frac{3 \mu^{2}\left(a q_{1}\right)^{\frac{2}{3}}}{2(1-\mu)\left(a q_{2}\right)^{\frac{2}{3}}}-\frac{15}{4\left(a q_{2}\right)^{\frac{2}{3}}}+\frac{15}{8\left(a q_{1}\right)^{\frac{2}{3}}\left(a q_{2}\right)^{\frac{2}{3}}}+\frac{15\left(a q_{1}\right)^{\frac{2}{3}}}{8\left(a q_{2}\right)^{\frac{2}{3}}}-\frac{15(1-\mu)\left(a q_{2}\right)^{\frac{4}{3}}}{16\left(a q_{1}\right)^{\frac{2}{3}}}-\frac{15(1-\mu)\left(a q_{2}\right)}{8\left(a q_{1}\right)^{\frac{2}{3}}} \\
& E_{42}=-\frac{15(1-\mu)\left(a q_{2}\right)^{\frac{1}{3}}}{16\left(a q_{1}\right)^{\frac{2}{3}}}+\frac{15\left(a q_{2}\right)^{\frac{2}{3}}}{16}-\frac{45 \mu\left(a q_{2}\right)^{\frac{2}{3}}}{32}+\frac{105 \mu\left(a q_{1}\right)^{\frac{4}{3}}}{16\left(a q_{2}\right)^{\frac{4}{3}}}-\frac{15 \mu\left(a q_{2}\right)^{\frac{1}{3}}}{16}-\frac{15 \mu}{16\left(a q_{2}\right)^{\frac{1}{3}}} \\
& +\frac{45 \mu\left(a q_{1}\right)^{\frac{2}{3}}}{16}-\frac{75 \mu}{64\left(a q_{2}\right)}-\frac{75 \mu\left(a q_{1}\right)^{2}}{16\left(a q_{2}\right)^{2}}+\frac{75 \mu\left(a q_{1}\right)^{\frac{2}{3}}}{16\left(a q_{2}\right)^{\frac{1}{3}}}+\frac{75 \mu\left(a q_{1}\right)^{\frac{2}{3}}}{32\left(a q_{2}\right)}-\frac{75 \mu\left(a q_{1}\right)^{\frac{4}{3}}}{32\left(a q_{2}\right)^{\frac{2}{3}}} \\
& E_{5}=\frac{5 M_{b}\left(2 r_{c}-1\right)}{4\left(r_{c}^{2}+T^{2}\right)^{\frac{3}{2}}}+\frac{3 M_{b}\left(\frac{1}{4}-\mu+\mu^{2}\right)}{4\left(r_{c}^{2}+T^{2}\right)^{\frac{5}{2}}} \\
& F_{0}=\frac{3(1-\mu)}{2}+\frac{3(1-\mu)\left(a q_{2}\right)^{\frac{2}{3}}}{2\left(a q_{1}\right)^{\frac{2}{3}}}-\frac{3(1-\mu)}{4\left(a q_{1}\right)^{\frac{2}{3}}}+\frac{3 \mu\left(a q_{1}\right)^{\frac{2}{3}}}{2\left(a q_{2}\right)^{\frac{2}{3}}}-\frac{3 \mu}{4\left(a q_{2}\right)^{\frac{2}{3}}} \\
& F_{01}=\frac{3 \mu}{2}+\left(\frac{-3(1-\mu)}{4\left(a q_{1}\right)^{\frac{2}{3}}}-\frac{3 \mu}{4\left(a q_{2}\right)^{\frac{2}{3}}}\right) e^{2} \\
& F_{11}=\frac{45}{16}-\frac{81 \mu}{16}-\frac{33 \mu\left(a q_{2}\right)^{\frac{2}{3}}}{16\left(a q_{1}\right)^{\frac{2}{3}}}+\frac{39 \mu\left(a q_{2}\right)^{\frac{2}{3}}}{16\left(a q_{1}\right)^{\frac{2}{3}}}+\frac{519}{32\left(a q_{1}\right)^{\frac{2}{3}}}-\frac{639 \mu}{32\left(a q_{1}\right)^{\frac{2}{3}}} \\
& +\frac{327\left(a q_{2}\right)^{\frac{2}{3}}}{16\left(a q_{1}\right)^{\frac{4}{3}}}-\frac{351 \mu\left(a q_{2}\right)^{\frac{2}{3}}}{16\left(a q_{1}\right)^{\frac{4}{3}}}-\frac{105(1-\mu)}{8\left(a q_{1}\right)^{\frac{4}{3}}}-\frac{15(1-\mu)}{16\left(a q_{1}\right)^{2}}+\frac{15(1-\mu)\left(a q_{2}\right)^{\frac{2}{3}}}{4\left(a q_{1}\right)^{2}}
\end{aligned}
$$




$$
\begin{aligned}
& F_{12}=\frac{33 \mu\left(a q_{1}\right)^{\frac{2}{3}}}{8\left(a q_{2}\right)^{\frac{2}{3}}}+\frac{9 \mu}{8\left(a q_{2}\right)^{\frac{2}{3}}}+\frac{375(1-\mu)\left(a q_{2}\right)^{\frac{2}{3}}}{32\left(a q_{1}\right)^{2}}+\frac{123\left(a q_{2}\right)^{\frac{4}{3}}}{16\left(a q_{1}\right)^{\frac{4}{3}}}-\frac{99 \mu\left(a q_{2}\right)^{\frac{4}{3}}}{16\left(a q_{1}\right)^{\frac{4}{3}}} \\
& -\frac{75(1-\mu)\left(a q_{2}\right)^{2}}{4\left(a q_{1}\right)^{2}}-\frac{15}{8\left(a q_{2}\right)^{\frac{2}{3}}}+\frac{15}{8\left(a q_{2}\right)}{ }^{\frac{2}{3}}\left(a q_{1}\right)^{\frac{2}{3}}+\frac{3\left(a q_{2}\right)^{\frac{2}{3}}}{2 \mu\left(a q_{1}\right)^{\frac{4}{3}}}+\frac{3\left(a q_{2}\right)^{\frac{2}{3}}}{2 \mu\left(a q_{1}\right)^{\frac{2}{3}}}-\frac{3\left(a q_{2}\right)^{\frac{4}{3}}}{2 \mu\left(a q_{1}\right)^{\frac{4}{3}}}-\frac{15\left(a q_{1}\right)^{\frac{2}{3}}}{4\left(a q_{2}\right)^{\frac{2}{3}}} \\
& F_{21}=\frac{-45}{16}+\frac{81 \mu}{16}+\frac{33 \mu\left(a q_{2}\right)^{\frac{2}{3}}}{16\left(a q_{1}\right)^{\frac{2}{3}}}-\frac{39 \mu\left(a q_{2}\right)^{\frac{2}{3}}}{16\left(a q_{1}\right)^{\frac{2}{3}}}-\frac{495}{32\left(a q_{1}\right)^{\frac{2}{3}}}+\frac{615}{32\left(a q_{1}\right)^{\frac{2}{3}}}-\frac{327\left(a q_{2}\right)^{\frac{2}{3}}}{16\left(a q_{1}\right)^{\frac{4}{3}}}+\frac{351 \mu\left(a q_{2}\right)^{\frac{2}{3}}}{16\left(a q_{1}\right)^{\frac{4}{3}}}+\frac{105(1-\mu)}{8\left(a q_{1}\right)^{\frac{4}{3}}} \\
& F_{22}=\frac{15(1-\mu)}{16\left(a q_{1}\right)^{2}}-\frac{15(1-\mu)\left(a q_{2}\right)^{\frac{2}{3}}}{4\left(a q_{1}\right)^{2}}-\frac{33 \mu\left(a q_{1}\right)^{\frac{2}{3}}}{8\left(a q_{2}\right)^{\frac{2}{3}}}-\frac{3 \mu}{8\left(a q_{2}\right)^{\frac{2}{3}}} \\
& -\frac{375(1-\mu)\left(a q_{2}\right)^{\frac{4}{3}}}{32\left(a q_{1}\right)^{2}}-\frac{123\left(a q_{2}\right)^{\frac{4}{3}}}{16\left(a q_{1}\right)^{\frac{4}{3}}}+\frac{99 \mu\left(a q_{2}\right)^{\frac{4}{3}}}{16\left(a q_{1}\right)^{\frac{4}{3}}}+\frac{75(1-\mu)\left(a q_{1}\right)^{2}}{16\left(a q_{1}\right)^{\frac{4}{3}}}+\frac{15}{8\left(a q_{2}\right)^{\frac{2}{3}}} \\
& -\frac{15 \mu}{8\left(a q_{2}\right)^{\frac{2}{3}}\left(a q_{1}\right)^{\frac{2}{3}}}+\frac{3\left(a q_{2}\right)^{\frac{2}{3}}}{2 \mu\left(a q_{1}\right)^{\frac{4}{3}}}-\frac{3\left(a q_{2}\right)^{\frac{2}{3}}}{2 \mu\left(a q_{1}\right)^{\frac{4}{3}}}-\frac{3\left(a q_{2}\right)^{\frac{2}{3}}}{2 \mu\left(a q_{1}\right)^{\frac{2}{3}}}+\frac{3\left(a q_{2}\right)^{\frac{4}{3}}}{2 \mu\left(a q_{1}\right)^{\frac{4}{3}}}+\frac{15\left(a q_{1}\right)^{\frac{2}{3}}}{4\left(a q_{2}\right)^{\frac{2}{3}}} \\
& F_{3}=-\frac{9}{4}+\frac{9 \mu}{64}+\frac{3\left(a q_{2}\right)^{\frac{2}{3}}}{8\left(a q_{1}\right)^{\frac{2}{3}}}-\frac{33 \mu\left(a q_{2}\right)^{\frac{2}{3}}}{8\left(a q_{1}\right)^{\frac{2}{3}}}+\frac{33}{16\left(a q_{1}\right)^{\frac{2}{3}}}-\frac{63 \mu}{16\left(a q_{1}\right)^{\frac{2}{3}}}-\frac{33 \mu\left(a q_{1}\right)^{\frac{2}{3}}}{64\left(a q_{2}\right)^{\frac{2}{3}}} \\
& +\frac{654 \mu}{32\left(a q_{2}\right)^{\frac{2}{3}}}+\frac{525 \mu\left(a q_{1}\right)^{\frac{2}{3}}}{32\left(a q_{2}\right)^{\frac{4}{3}}}+\frac{225 \mu}{64\left(a q_{2}\right)^{\frac{4}{3}}}-\frac{15 \mu}{16\left(a q_{2}\right)^{2}}+\frac{15 \mu\left(a q_{1}\right)^{\frac{2}{3}}}{4\left(a q_{2}\right)^{2}}+\frac{3 \mu\left(a q_{2}\right)^{-\frac{2}{3}}}{4\left(a q_{1}\right)^{\frac{1}{3}}}-\frac{15 \mu\left(a q_{2}\right)^{-\frac{2}{3}}}{8\left(a q_{1}\right)} \\
& +\frac{3 \mu^{2}\left(a q_{1}\right)^{\frac{2}{3}}}{2(1-\mu)\left(a q_{2}\right)^{\frac{4}{3}}}-\frac{3 \mu^{2}\left(a q_{1}\right)^{\frac{1}{3}}}{2(1-\mu)\left(a q_{2}\right)^{\frac{4}{3}}}+\frac{3 \mu^{2}\left(a q_{1}\right)^{\frac{2}{3}}}{2(1-\mu)\left(a q_{2}\right)^{\frac{2}{3}}}+\frac{15 \mu}{4\left(a q_{1}\right)}-\frac{15}{4\left(a q_{2}\right)^{\frac{2}{3}}}+\frac{15}{8\left(a q_{1}\right)^{\frac{2}{3}}\left(a q_{2}\right)^{\frac{2}{3}}}+\frac{15\left(a q_{1}\right)^{\frac{2}{3}}}{8\left(a q_{2}\right)^{\frac{2}{3}}} \\
& -\frac{15(1-\mu)\left(a q_{2}\right)^{\frac{4}{3}}}{16\left(a q_{1}\right)^{\frac{2}{3}}}-\frac{15(1-\mu)\left(a q_{2}\right)}{8\left(a q_{1}\right)^{\frac{2}{3}}}-\frac{15(1-\mu)\left(\mathrm{aq}_{2}\right)^{\frac{1}{3}}}{16\left(a q_{2}\right)^{\frac{2}{3}}}+\frac{15\left(a q_{2}\right)^{\frac{2}{3}}}{16}-\frac{75 \mu\left(a q_{1}\right)^{\frac{4}{3}}}{16\left(a q_{2}\right)^{\frac{4}{3}}}+\frac{75 \mu\left(a q_{1}\right)^{\frac{2}{3}}}{16\left(a q_{2}\right)^{\frac{1}{3}}} \\
& +\frac{75 \mu\left(a q_{1}\right)^{\frac{2}{3}}}{16\left(a q_{2}\right)}-\frac{75 \mu\left(a q_{1}\right)^{\frac{4}{3}}}{32\left(a q_{2}\right)^{\frac{2}{3}}}-\frac{15 \mu\left(a q_{2}\right)^{\frac{1}{3}}}{16}-\frac{15 \mu}{8\left(a q_{2}\right)^{\frac{1}{3}}}+\frac{15 \mu\left(a q_{1}\right)^{\frac{2}{3}}}{8}-\frac{75 \mu}{64\left(a q_{2}\right)} \\
& F_{4}=\frac{9}{4}-\frac{9 \mu}{64}-\frac{3\left(a q_{2}\right)^{\frac{2}{3}}}{8\left(a q_{1}\right)^{\frac{2}{3}}}+\frac{33 \mu\left(a q_{2}\right)^{\frac{2}{3}}}{8\left(a q_{1}\right)^{\frac{2}{3}}}-\frac{21}{16\left(a q_{1}\right)^{\frac{2}{3}}}+\frac{51 \mu}{16\left(a q_{1}\right)^{\frac{2}{3}}}+\frac{33 \mu\left(\mathrm{aq}_{1}\right)^{\frac{2}{3}}}{64\left(a q_{2}\right)^{\frac{2}{3}}}-\frac{630 \mu}{32\left(a q_{2}\right)^{\frac{2}{3}}} \\
& -\frac{525 \mu\left(a q_{1}\right)^{\frac{2}{3}}}{32\left(a q_{2}\right)^{\frac{4}{3}}}-\frac{225 \mu}{64\left(a q_{2}\right)^{\frac{4}{3}}}+\frac{15 \mu}{16\left(a q_{2}\right)^{2}}-\frac{15 \mu\left(a q_{1}\right)^{\frac{2}{3}}}{4\left(a q_{2}\right)^{2}}-\frac{3 \mu^{2}\left(a q_{2}\right)^{-\frac{2}{3}}}{4\left(a q_{1}\right)^{\frac{1}{3}}}+\frac{15 \mu\left(a q_{2}\right)^{-\frac{2}{3}}}{8\left(a q_{1}\right)}-\frac{3 \mu^{2}\left(a q_{1}\right)^{\frac{2}{3}}}{2(1-\mu)\left(a q_{2}\right)^{\frac{4}{3}}} \\
& +\frac{3 \mu^{2}\left(a q_{1}\right)^{\frac{1}{3}}}{2(1-\mu)\left(a q_{2}\right)^{\frac{4}{3}}}-\frac{3 \mu^{2}\left(a q_{1}\right)^{\frac{2}{3}}}{2(1-\mu)\left(a q_{2}\right)^{\frac{2}{3}}}-\frac{15 \mu}{4\left(a q_{1}\right)}+\frac{15}{4\left(a q_{2}\right)^{\frac{2}{3}}}-\frac{15}{8\left(a q_{1}\right)^{\frac{2}{3}}\left(a q_{2}\right)^{\frac{2}{3}}}-\frac{15\left(a q_{1}\right)^{\frac{2}{3}}}{8\left(a q_{2}\right)^{\frac{2}{3}}}+\frac{15(1-\mu)\left(a q_{2}\right)^{\frac{4}{3}}}{16\left(a q_{1}\right)^{\frac{2}{3}}} \\
& +\frac{15(1-\mu)\left(a q_{2}\right)}{8\left(a q_{1}\right)^{\frac{2}{3}}}+\frac{15(1-\mu)\left(a q_{2}\right)^{\frac{1}{3}}}{16\left(a q_{1}\right)^{\frac{2}{3}}}-\frac{15\left(a q_{2}\right)^{\frac{2}{3}}}{16}-\frac{375 \mu\left(a q_{1}\right)^{\frac{4}{3}}}{32\left(a q_{2}\right)^{2}}+\frac{75 \mu\left(a q_{1}\right)^{2}}{32\left(a q_{2}\right)^{2}}-\frac{75 \mu\left(a q_{1}\right)^{\frac{4}{3}}}{16\left(a q_{2}\right)^{\frac{4}{3}}}-\frac{75 \mu\left(a q_{1}\right)^{\frac{2}{3}}}{32\left(a q_{2}\right)^{\frac{1}{3}}} \\
& -\frac{75 \mu\left(a q_{1}\right)^{\frac{2}{3}}}{32\left(a q_{2}\right)}+\frac{75 \mu\left(a q_{1}\right)^{\frac{4}{3}}}{32\left(a q_{2}\right)^{\frac{2}{3}}}+\frac{15 \mu\left(a q_{2}\right)^{\frac{2}{3}}}{32}-\frac{15 \mu\left(a q_{2}\right)^{\frac{1}{3}}}{16}+\frac{15 \mu}{8\left(a q_{2}\right)^{\frac{1}{3}}}-\frac{15 \mu\left(a q_{1}\right)^{\frac{2}{3}}}{8}+\frac{75 \mu}{64\left(a q_{2}\right)} \\
& F_{5}=\frac{7 M_{b}\left(2 r_{c}-1\right)}{4\left(r_{c}^{2}+T^{2}\right)^{\frac{3}{2}}}+\frac{9 M_{b}}{4\left(r_{c}^{2}+T^{2}\right)^{\frac{5}{2}}} \\
& G_{0}=\frac{3(1-\mu)}{2\left(a q_{1}\right)^{\frac{2}{3}}}+\frac{3(1-\mu)}{2}-\frac{3(1-\mu)\left(a q_{2}\right)^{\frac{2}{3}}}{4\left(a q_{1}\right)^{\frac{2}{3}}}-\frac{3 \mu}{2\left(a q_{1}\right)^{\frac{2}{3}}}-\frac{3 \mu\left(a q_{1}\right)^{\frac{2}{3}}}{2\left(a q_{2}\right)^{\frac{2}{3}}}-\frac{3 \mu}{2}+\left(\frac{3(1-\mu)}{2\left(a q_{1}\right)^{\frac{2}{3}}}-\frac{3 \mu}{2\left(a q_{2}\right)^{\frac{2}{3}}}\right) e^{2}
\end{aligned}
$$




$$
\begin{aligned}
& G_{1}=\frac{21}{16\left(a q_{1}\right)^{\frac{2}{3}}}-\frac{81 \mu}{16\left(a q_{1}\right)^{\frac{2}{3}}}+\frac{87}{16}-\frac{123 \mu}{16}+\frac{81\left(a q_{2}\right)^{\frac{2}{3}}}{16\left(a q_{1}\right)^{\frac{2}{3}}}-\frac{27\left(a q_{2}\right)^{\frac{2}{3}}}{16\left(a q_{1}\right)^{\frac{2}{3}}}+\frac{15(1-\mu)}{4\left(a q_{1}\right)^{\frac{4}{3}}}-\frac{3 \mu\left(a q_{2}\right)^{\frac{2}{3}}}{8\left(a q_{1}\right)^{\frac{4}{3}}} \\
& -\frac{45(1-\mu)\left(a q_{2}\right)^{\frac{2}{3}}}{8\left(a q_{1}\right)^{2}}+\frac{15(1-\mu)}{8\left(a q_{1}\right)^{2}}-\frac{15(1-\mu)\left(a q_{2}\right)^{\frac{4}{3}}}{16\left(a q_{1}\right)^{2}}-\frac{9 \mu}{4\left(a q_{2}\right)^{\frac{2}{3}}}+\frac{33 \mu\left(a q_{1}\right)^{\frac{2}{3}}}{8\left(a q_{2}\right)^{\frac{2}{3}}}-\frac{183\left(a q_{2}\right)^{\frac{4}{3}}}{16\left(a q_{1}\right)^{\frac{4}{3}}}+\frac{159 \mu\left(a q_{2}\right)^{\frac{4}{3}}}{16\left(a q_{1}\right)^{\frac{4}{3}}} \\
& +\frac{75(1-\mu)\left(a q_{2}\right)^{2}}{16\left(a q_{1}\right)^{2}}-\frac{15}{8\left(a q_{1}\right)^{\frac{2}{3}}\left(a q_{2}\right)^{\frac{2}{3}}}-\frac{3\left(a q_{2}\right)^{\frac{2}{3}}}{2 \mu\left(a q_{1}\right)^{\frac{4}{3}}}-\frac{3\left(a q_{2}\right)^{\frac{2}{3}}}{2 \mu\left(a q_{1}\right)^{\frac{2}{3}}}+\frac{3\left(a q_{2}\right)^{\frac{4}{3}}}{2 \mu\left(a q_{1}\right)^{\frac{4}{3}}}+\frac{15}{4\left(a q_{2}\right)^{\frac{2}{3}}\left(a q_{1}\right)^{\frac{2}{3}}}-\frac{15\left(a q_{1}\right)^{\frac{2}{3}}}{4\left(a q_{2}\right)^{\frac{2}{3}}} \\
& G_{2}=\frac{27}{16\left(a q_{1}\right)^{\frac{2}{3}}}+\frac{33 \mu}{16\left(a q_{1}\right)^{\frac{2}{3}}}-\frac{87}{16}+\frac{123 \mu}{16}-\frac{81\left(a q_{2}\right)^{\frac{2}{3}}}{16\left(a q_{1}\right)^{\frac{2}{3}}}+\frac{27\left(a q_{2}\right)^{\frac{2}{3}}}{16\left(a q_{1}\right)^{\frac{2}{3}}}-\frac{15(1-\mu)}{4\left(a q_{1}\right)^{\frac{4}{3}}}-\frac{9\left(a q_{2}\right)^{\frac{2}{3}}}{8\left(a q_{1}\right)^{\frac{4}{3}}}-\frac{3 \mu\left(a q_{2}\right)^{\frac{2}{3}}}{8\left(a q_{1}\right)^{\frac{4}{3}}} \\
& +\frac{45(1-\mu)\left(a q_{2}\right)^{\frac{2}{3}}}{8\left(a q_{1}\right)^{2}}-\frac{15(1-\mu)}{8\left(a q_{1}\right)^{2}}+\frac{15(1-\mu)\left(a q_{2}\right)^{\frac{4}{3}}}{16\left(a q_{1}\right)^{2}}+\frac{9 \mu}{4\left(a q_{2}\right)^{\frac{2}{3}}}-\frac{33 \mu\left(a q_{1}\right)^{\frac{2}{3}}}{8\left(a q_{2}\right)^{\frac{2}{3}}}+\frac{183\left(a q_{2}\right)^{\frac{4}{3}}}{16\left(a q_{1}\right)^{\frac{4}{3}}}-\frac{159 \mu\left(a q_{2}\right)^{\frac{4}{3}}}{16\left(a q_{1}\right)^{\frac{4}{3}}} \\
& -\frac{75(1-\mu)\left(a q_{2}\right)^{2}}{16\left(a q_{1}\right)^{2}}+\frac{15}{8\left(a q_{1}\right)^{\frac{2}{3}}\left(a q_{2}\right)^{\frac{2}{3}}}+\frac{3\left(a q_{2}\right)^{\frac{2}{3}}}{2 \mu\left(a q_{1}\right)^{\frac{4}{3}}}+\frac{3\left(a q_{2}\right)^{\frac{2}{3}}}{2 \mu\left(a q_{1}\right)^{\frac{2}{3}}}-\frac{3\left(a q_{2}\right)^{\frac{4}{3}}}{2 \mu\left(a q_{1}\right)^{\frac{4}{3}}}-\frac{15}{4\left(a q_{2}\right)^{\frac{2}{3}}\left(a q_{1}\right)^{\frac{2}{3}}}+\frac{15\left(a q_{1}\right)^{\frac{2}{3}}}{4\left(a q_{2}\right)^{\frac{2}{3}}} \\
& G_{3}=\frac{-9}{16\left(a q_{1}\right)^{\frac{2}{3}}}+\frac{9 \mu}{16\left(a q_{1}\right)^{\frac{2}{3}}}+\frac{9}{4}-\frac{129 \mu}{32}-\frac{3\left(a q_{2}\right)^{\frac{2}{3}}}{8\left(a q_{1}\right)^{\frac{2}{3}}}+\frac{33 \mu\left(a q_{2}\right)^{\frac{2}{3}}}{8\left(a q_{1}\right)^{\frac{2}{3}}}-\frac{147 \mu}{32\left(a q_{2}\right)^{\frac{2}{3}}}-\frac{3 \mu\left(\mathrm{aq}_{1}\right)^{\frac{2}{3}}}{32\left(a q_{2}\right)^{\frac{2}{3}}} \\
& +\frac{105 \mu}{32\left(a q_{2}\right)^{\frac{4}{3}}}-\frac{165 \mu\left(a q_{1}\right)^{\frac{2}{3}}}{32\left(a q_{2}\right)^{\frac{4}{3}}}+\frac{45 \mu\left(a q_{1}\right)^{\frac{2}{3}}}{8\left(a q_{2}\right)^{2}}-\frac{15 \mu}{8\left(a q_{2}\right)^{2}}+\frac{15 \mu\left(a q_{1}\right)^{\frac{4}{3}}}{16\left(a q_{2}\right)^{2}}+\frac{9 \mu\left(a q_{2}\right)^{\frac{-2}{3}}}{4\left(a q_{1}\right)^{\frac{1}{3}}}+\frac{15 \mu\left(a q_{2}\right)^{\frac{-2}{3}}}{4\left(a q_{1}\right)}-\frac{15 \mu}{4\left(a q_{1}\right)} \\
& +\frac{3 \mu^{2}\left(a q_{1}\right)^{\frac{2}{3}}}{4(1-\mu)\left(a q_{2}\right)^{\frac{4}{3}}}-\frac{3 \mu^{2}\left(a q_{1}\right)^{\frac{1}{3}}}{4(1-\mu)\left(a q_{2}\right)^{\frac{4}{3}}}+\frac{3 \mu^{2}\left(a q_{1}\right)^{\frac{2}{3}}}{4(1-\mu)\left(a q_{2}\right)^{\frac{2}{3}}}+\frac{15}{4\left(a q_{2}\right)^{\frac{2}{3}}}-\frac{15}{8\left(a q_{2}\right)^{\frac{2}{3}}\left(a q_{1}\right)^{\frac{2}{3}}}-\frac{15 \mu}{8\left(a q_{2}\right)^{\frac{2}{3}}\left(a q_{1}\right)^{\frac{2}{3}}} \\
& -\frac{15\left(a q_{1}\right)^{\frac{2}{3}}}{8\left(a q_{2}\right)^{\frac{2}{3}}}+\frac{15(1-\mu)\left(a q_{2}\right)^{\frac{4}{3}}}{16\left(a q_{1}\right)^{\frac{2}{3}}}+\frac{15(1-\mu)\left(a q_{2}\right)}{8\left(a q_{1}\right)^{\frac{2}{3}}}+\frac{15(1-\mu)\left(a q_{2}\right)^{\frac{1}{3}}}{16\left(a q_{1}\right)^{\frac{2}{3}}}-\frac{15\left(a q_{2}\right)^{\frac{2}{3}}}{16}-\frac{15 \mu\left(a q_{2}\right)^{\frac{2}{3}}}{32}-\frac{195 \mu}{32\left(a q_{2}\right)^{\frac{1}{3}}} \\
& -\frac{75 \mu}{32\left(a q_{2}\right)}-\frac{75 \mu\left(a q_{1}\right)^{2}}{16\left(a q_{2}\right)^{2}}+\frac{135 \mu\left(a q_{1}\right)^{\frac{4}{3}}}{16\left(a q_{2}\right)^{\frac{4}{3}}}+\frac{75 \mu\left(a q_{1}\right)^{\frac{2}{3}}}{32\left(a q_{2}\right)^{\frac{1}{3}}}+\frac{75 \mu\left(a q_{1}\right)^{\frac{2}{3}}}{32\left(a q_{2}\right)}-\frac{75 \mu\left(a q_{1}\right)^{\frac{4}{3}}}{2\left(a q_{2}\right)^{\frac{2}{3}}} \frac{15 \mu\left(\mathrm{aq}_{1}\right)^{\frac{2}{3}}}{4}-\frac{45 \mu\left(a q_{2}\right)^{\frac{1}{3}}}{16} \\
& G_{4}=\frac{9}{16\left(a q_{1}\right)^{\frac{2}{3}}}-\frac{9 \mu}{16\left(a q_{1}\right)^{\frac{2}{3}}}-\frac{9}{4}+\frac{201 \mu}{32}+\frac{3\left(a q_{2}\right)^{\frac{2}{3}}}{8\left(a q_{1}\right)^{\frac{2}{3}}}-\frac{33 \mu\left(a q_{2}\right)^{\frac{2}{3}}}{8\left(a q_{1}\right)^{\frac{2}{3}}}+\frac{51 \mu}{32\left(a q_{2}\right)^{\frac{2}{3}}}+\frac{3 \mu\left(a q_{1}\right)^{\frac{2}{3}}}{32\left(a q_{2}\right)^{\frac{4}{3}}}-\frac{105 \mu}{32\left(a q_{2}\right)^{\frac{4}{3}}} \\
& +\frac{165 \mu\left(a q_{1}\right)^{\frac{2}{3}}}{32\left(a q_{2}\right)^{\frac{4}{3}}}-\frac{45 \mu\left(a q_{1}\right)^{\frac{2}{3}}}{8\left(a q_{2}\right)^{2}}+\frac{15 \mu}{8\left(a q_{2}\right)^{2}}-\frac{15 \mu\left(a q_{1}\right)^{\frac{4}{3}}}{16\left(a q_{2}\right)^{2}}-\frac{9 \mu\left(a q_{2}\right)^{\frac{-2}{3}}}{4\left(a q_{1}\right)^{\frac{1}{3}}}-\frac{15 \mu\left(a q_{2}\right)^{\frac{-2}{3}}}{4\left(a q_{1}\right)}+\frac{15 \mu}{4\left(a q_{1}\right)}-\frac{3 \mu^{2}\left(a q_{1}\right)^{\frac{2}{3}}}{4(1-\mu)\left(a q_{2}\right)^{\frac{4}{3}}} \\
& +\frac{3 \mu^{2}\left(a q_{1}\right)^{\frac{1}{3}}}{4(1-\mu)\left(a q_{2}\right)^{\frac{4}{3}}}-\frac{3 \mu^{2}\left(a q_{1}\right)^{\frac{2}{3}}}{4(1-\mu)\left(a q_{2}\right)^{\frac{2}{3}}}-\frac{15}{4\left(a q_{2}\right)^{\frac{2}{3}}}+\frac{15}{8\left(a q_{2}\right)^{\frac{2}{3}}\left(a q_{1}\right)^{\frac{2}{3}}}+\frac{15 \mu}{8\left(a q_{2}\right)^{\frac{2}{3}}\left(a q_{1}\right)^{\frac{2}{3}}}+\frac{15\left(a q_{1}\right)^{\frac{2}{3}}}{8\left(a q_{2}\right)^{\frac{2}{3}}} \\
& -\frac{15(1-\mu)\left(a q_{2}\right)^{\frac{4}{3}}}{16\left(a q_{1}\right)^{\frac{2}{3}}}-\frac{15(1-\mu)\left(a q_{2}\right)}{8\left(a q_{1}\right)^{\frac{2}{3}}}-\frac{15(1-\mu)\left(a q_{1}\right)^{\frac{1}{3}}}{16\left(a q_{1}\right)^{\frac{2}{3}}}+\frac{15\left(a q_{2}\right)^{\frac{2}{3}}}{16}+\frac{15 \mu\left(a q_{2}\right)^{\frac{2}{3}}}{32}+\frac{195 \mu}{32\left(a q_{2}\right)^{\frac{1}{3}}}+\frac{75 \mu}{32\left(a q_{2}\right)} \\
& +\frac{75 \mu\left(a q_{1}\right)^{2}}{16\left(a q_{1}\right)^{2}}-\frac{135 \mu\left(a q_{1}\right)^{\frac{4}{3}}}{16\left(a q_{2}\right)^{\frac{4}{3}}}-\frac{75 \mu\left(a q_{1}\right)^{\frac{2}{3}}}{16\left(a q_{2}\right)^{\frac{1}{3}}}-\frac{75 \mu\left(a q_{1}\right)^{\frac{2}{3}}}{32\left(a q_{2}\right)}+\frac{75 \mu\left(a q_{1}\right)^{\frac{4}{3}}}{32\left(a q_{2}\right)^{\frac{2}{3}}}-\frac{15 \mu\left(a q_{1}\right)^{\frac{2}{3}}}{4}+\frac{45 \mu\left(a q_{2}\right)^{\frac{1}{3}}}{16} \\
& G_{5}=-\frac{11 M_{b}\left(2 r_{c}-1\right)}{6\left(r_{c}^{2}+T^{2}\right)^{\frac{3}{2}}}+\frac{3 M_{b}\left(\frac{1}{2}-\mu\right)}{2\left(r_{c}^{2}+T^{2}\right)^{\frac{5}{2}}}
\end{aligned}
$$

Substituting the values of (14) into the characteristic equation (13) and restricting ourselves only to the linear term in $\mathrm{M}_{b}$, $\mathrm{a}, e^{2}, \sigma_{1}, \sigma_{2}, \sigma_{3}, \sigma_{4}, \alpha, q_{1}$, and $q_{2}$ where $\alpha=1-\alpha, q_{1}=1-\beta_{1}$ and $q_{2}=1-\beta_{2}$ and neglecting the second and higher order terms of $M_{\mathrm{b}}, e^{2}, \beta_{1}, \beta_{2}, \alpha, \sigma_{1}, \sigma_{2}, \sigma_{3}$, and $\sigma_{4}$ and their products we obtain:

$$
\left.4\left(\lambda^{2}\right)^{2}+4\left(4-3 \phi_{1}\right) \lambda^{2}+27 \mu(1-\mu)\right)+4 \phi_{2}=0
$$


Where;

$$
\left.\begin{array}{l}
\phi_{1}=\left(1-e^{2}\right)^{-\frac{1}{2}}\left[1+\frac{175(1-\mu)}{8} \sigma_{1}-\frac{167(1-\mu)}{8} \sigma_{2}+\frac{125 \mu}{8} \sigma_{3}-\frac{117 \mu}{8} \sigma_{4}\right]+\frac{M_{b}\left(2 r_{c}+3\right)}{\left(r_{c}^{2}+T^{2}\right)^{\frac{3}{2}}}-\frac{3 M_{b} r_{c}^{2}}{\left(r_{c}^{2}+T^{2}\right)^{\frac{5}{2}}} \\
\phi_{2}=3 \mu(1-\mu) \alpha+\frac{3 \mu(1-\mu)}{2}\left(\beta_{1}+\beta_{2}\right)+\frac{45 \mu(1-\mu)}{4} e^{2}+H_{1}+H_{2}+H_{3}+H_{4}+H_{0}
\end{array}\right\}
$$

Where

$$
\begin{aligned}
& H_{1}=\left(\frac{333(1-\mu)}{4}-\frac{2547 \mu(1-\mu)}{32}+\frac{1575 \mu}{16}-\frac{3087 \mu^{2}}{32}+\frac{207(1-\mu)^{2}}{32}\right) \sigma_{1} \\
& H_{2}=\left(\frac{-675(1-\mu)}{8}+\frac{2475 \mu(1-\mu)}{32}-\frac{1377 \mu}{16}+\frac{2691 \mu^{2}}{32}-\frac{171(1-\mu)^{2}}{32}\right) \sigma_{2} \\
& H_{3}=\left(\frac{-297(1-\mu)}{64}+\frac{13221 \mu(1-\mu)}{256}+\frac{693 \mu^{2}}{64}+\frac{765 \mu}{64}+\frac{171(1-\mu)^{2}}{32}\right) \sigma_{3} \\
& H_{4}=\left(\frac{225(1-\mu)}{64}-\frac{1034 \mu(1-\mu)}{256}-\frac{621 \mu^{2}}{64}-\frac{837 \mu}{64}-\frac{135(1-\mu)^{2}}{32}\right) \sigma_{4} \\
& H_{0}=\frac{33 M_{b}\left(2 r_{c}-1\right)}{2\left(r_{c}^{2}+T^{2}\right)^{\frac{3}{2}}}+\frac{27 M_{b}}{4\left(r_{c}^{2}+T^{2}\right)^{\frac{5}{2}}}
\end{aligned}
$$

equation (15) is a quadratic equation in $\lambda^{2}$ which yield

$$
\lambda^{2}=-\frac{\left(4-3 \phi_{1}\right) \pm\left[\left(4-3 \phi_{1}\right)^{2}-27 \mu(1-\mu)-4 \phi_{2}\right]^{\frac{1}{2}}}{2}
$$

For the motion to be stable, we require $\lambda$ to be pure imaginary i.e the motion of the particle must be bounded and periodic, therefore we choose $\mu, \phi_{1}, \phi_{2}$ such that $\lambda^{2}<0$, we have $3 \phi_{1}-4 \leq 0$ and the discriminant

$$
\Delta=\left(4-3 \phi_{1}\right)^{2}-27 \mu(1-\mu)-4 \phi_{2}>0
$$

which yields

$$
0<e \leq\left[1-\frac{9}{16}\left(1+\frac{175(1-\mu)}{8} \sigma_{1}-\frac{167(1-\mu)}{8} \sigma_{2}+\frac{125 \mu}{8} \sigma_{3}-\frac{117 \mu}{8} \sigma_{4}\right)^{2}\right]^{\frac{1}{2}}+\frac{M_{b}\left(2 r_{c}+3\right)}{\left(r_{c}^{2}+T^{2}\right)^{\frac{3}{2}}}-\frac{3 M_{b} r_{c}^{2}}{\left(r_{c}^{2}+T^{2}\right)^{\frac{5}{2}}}
$$

When $M_{b}=\sigma_{1}=\sigma_{2}=\sigma_{3}=\sigma_{4}=0$ (19) becomes

$$
0<e \leq \frac{\sqrt{7}}{4}
$$

If (18) is not satisfied, the roots will be either real or complex conjugate. In case they are complex roots, the positive real part indicates instability of the equilibrium points being investigated.

The characteristic root obtained from (15) is thus:

$$
\lambda_{1,2}^{2}=\left(1-\frac{3}{2} e^{2}-\frac{525(1-\mu)}{8} \sigma_{1}+\frac{501(1-\mu)}{8} \sigma_{2}-\frac{375 \mu}{8} \sigma_{3}+\frac{351 \mu}{8} \sigma_{4}-\frac{3 M_{b}\left(2 r_{c+} 3\right)}{\left(r_{c}^{2}+T^{2}\right)^{\frac{3}{2}}}+\frac{9 M_{b} r_{c}^{2}}{\left(r_{c}^{2}+T^{2}\right)^{\frac{5}{2}}}\right) \pm K
$$

where

$$
\begin{aligned}
K & =\left[K_{1}-K_{2}+K_{3}\right]^{\frac{1}{2}} \\
K_{1} & =\left(K_{0}-\frac{333}{8} \sigma_{1}-\frac{45}{8} \sigma_{2}+\frac{9081}{64} \sigma_{3}-\frac{6777}{64} \sigma_{4}+\frac{66 M_{b}\left(2 r_{c-1} 1\right)}{\left(r_{c}^{2}+T^{2}\right)^{\frac{3}{2}}}+\frac{27 M_{b}}{\left(r_{c}^{2}+T^{2}\right)^{\frac{5}{2}}}\right) \mu^{2}
\end{aligned}
$$




$$
\begin{aligned}
& K_{0}=\left(27+12 \alpha+6 \beta_{1}+6 \beta_{2}+45 e^{2}\right) \\
& K_{2}=\left(K_{0}-\frac{3525}{8} \sigma_{1}+\frac{3765}{8} \sigma_{2}+\frac{20493}{64} \sigma_{3}-\frac{17805}{64} \sigma_{4}+\frac{66 M_{b}\left(2 r_{c-} 1\right)}{\left(r_{c}^{2}+T^{2}\right)^{\frac{3}{2}}}+\frac{108 M_{b}}{\left(r_{c}^{2}+T^{2}\right)^{\frac{5}{2}}}\right) \mu+ \\
& K_{3}=1-3 e^{2}-\frac{3921}{8} \sigma_{1}+\frac{3873}{8} \sigma_{2}-\frac{45}{16} \sigma_{3}+\frac{45}{16} \sigma_{4}+\frac{2 M_{b}\left(2 r_{c+} 3\right)}{\left(r_{c}^{2}+T^{2}\right)^{\frac{3}{2}}}-\frac{6 M_{b} r_{c}{ }^{2}}{\left(r_{c}^{2}+T^{2}\right)^{\frac{5}{2}}}
\end{aligned}
$$

In order to examine the effect of binary system parameters on the stability of triangular equilibrium points we have used (21) to compute the characteristic root for the binary system(xi-Bootis) in Table 7 and Table 8, with the mass ratio in the range $0.0005 \leq \mu \leq 0.11942$. In Table 7,the roots are pure imaginary numbers for values of $\mu, 0.0005 \leq \mu \leq 0.03852$ indicating stability of the system dynamics due the effect of the mass ratio and the disc.However,in Table 8 complex roots are obtain due to the combine effect of radition pressure,triaxiality and the disc which has caused instability to the triangular equilibrium point.

From (18) we have:

$$
\begin{aligned}
\Delta & =\left(27+12 a+6 \beta_{1}+6 \beta_{2}+45 e^{2}\right) \mu^{2}+\left(\frac{333}{8} \sigma_{1}-\frac{45}{8} \sigma_{2}+\frac{9081}{64} \sigma_{3}-\frac{6777}{64} \sigma_{4}\right) \mu^{2} \\
& +\left(\frac{66 M_{b}\left(2 r_{c}-1\right)}{\left(r_{c}^{2}+T^{2}\right)^{\frac{3}{2}}}+\frac{27 M_{b}}{\left(r_{c}^{2}+T^{2}\right)^{\frac{5}{2}}}\right) \mu^{2}-\left(27+12 a+6 \beta_{1}+6 \beta_{2}+45 e^{2}-\frac{3525}{8} \sigma_{1}+\frac{3765}{8} \sigma_{2}+\frac{20493}{64} \sigma_{3}-\frac{17805}{64} \sigma_{4}\right) \mu \\
& +\left(\frac{66 M_{b}\left(2 r_{c}-1\right)}{\left(r_{c}^{2}+T^{2}\right)^{\frac{3}{2}}}+\frac{108 M_{b}}{\left(r_{c}^{2}+T^{2}\right)^{\frac{5}{2}}}\right) \mu+\left(1-3 e^{2}-\frac{3921}{8} \sigma_{1}+\frac{3873}{8} \sigma_{2}-\frac{45}{16} \sigma_{3}+\frac{45}{1664805} \sigma_{4}\right) \\
& +\frac{2 M_{b}\left(2 r_{c}+3\right)}{\left(r_{c}^{2}+T^{2}\right)^{\frac{3}{2}}}-\frac{6 M_{b} r_{c}^{2}}{\left(r_{c}^{2}+T^{2}\right)^{\frac{5}{2}}}
\end{aligned}
$$

Equations (18),(20) and (22) gives the necessary condition for the stability of triangular equilibrium points. The solution of the quadratic equation $\Delta=0$ when the disriminant vanishes for $\mu$ gives the critical value $\mu_{c}$ of the mass parameter given by:

$$
\begin{aligned}
\mu_{C} & =\frac{1}{2}\left(1-\sqrt{\frac{23}{27}}\right)-\frac{4}{27 \sqrt{69}} a-\frac{2}{27 \sqrt{69}} \beta_{1}-\frac{2}{27 \sqrt{69}} \beta_{2}-\frac{14 e^{2}}{9 \sqrt{69}}+\left(\frac{-643}{72}-\frac{6013}{72 \sqrt{69}}\right) \sigma_{1}+\left(\frac{635}{72}+\frac{5909}{27 \sqrt{69}}\right) \sigma_{2} \\
& +\frac{1}{32}\left(\frac{317}{37279}-\frac{18667}{18 \sqrt{69}}\right) \sigma_{3}-\frac{1}{32}\left(\frac{919}{9319677279}-\frac{5945}{6 \sqrt{69}}\right) \sigma_{4}+\left(\frac{3}{2}+\frac{\left(76-8 r_{c}\right)\left(r_{c}^{2}+T^{2}\right)}{27 \sqrt{ } 69}-\frac{83+12 r_{c}^{2}}{6 \sqrt{ } 69}\right) \\
& \times \frac{M_{b}}{\left(r_{c}^{2}+T^{2}\right)^{\frac{5}{2}}}
\end{aligned}
$$

We have used equation (23) to study the effects of eccentricity, triaxiality, and radiation pressure, and the potential from the belt on the critical mass value $\mu_{c}$ by using the values of $e, \alpha, \beta_{1}, \beta_{2}$ of the binary system and allocating arbitrary values to the triaxiality factors $\sigma_{i}(\mathrm{i}=1,2,3,4)$. The critical mass parameters $\mu_{c}$ indicates the effects of the various parameters on the size of region of stability. We rewrite Eq.(21) as

$$
\lambda_{1,2}^{2}=\frac{-B \pm \sqrt{\Delta}}{2}
$$

where

$$
B=1-\frac{3}{2} e^{2}-\frac{525(1-\mu)}{8} \sigma_{1}+\frac{501(1-\mu)}{8} \sigma_{2}-\frac{375 \mu}{8} \sigma_{3}+\frac{375 \mu}{8} \sigma_{4}-\frac{3 M_{b}\left(2 r_{c+} 3\right)}{\left(r_{c}^{2}+T^{2}\right)^{\frac{3}{2}}}+\frac{9 M_{b} r_{c}{ }^{2}}{\left(r_{c}^{2}+T^{2}\right)^{\frac{5}{2}}}>0
$$

The roots are the functions of the values of the mass parameter $\mu$,the radiation, triaxiality and the belt.Hence the nature of these roots depends upon the nature of the discriminant $\Delta$ and B.since B $>0, \Delta>0$ in the interval $0<\mu<\mu_{c}$, thefore the roots of (24) $\lambda_{i}(i=1,2,3,4)$ are distinct pure imaginary numbers. Consequently the triangular points are stable in this region. $\lambda_{1,2,3,4}= \pm i \Lambda_{n}(\mathrm{n}=1,2)$ where $\Lambda_{n}=\sqrt{\frac{1}{2}}(-\mathbf{B} \pm \sqrt{\Delta}), \mathrm{n}=1,2$ and If $\mu_{c}<\mu<\frac{1}{2}, \Delta<0$ the real parts of two of the roots of (24) are positive .Hence the triangular points is unstable. When $\mu=\mu_{c}, \Delta=0$ the roots are double roots, which induces instability at the points. Therefore, the triangular points are stable for $0<\mu<\mu_{c}$ and unstable for $\mu_{c}<\mu<\frac{1}{2}, \mu_{c}$ is the critical 
mass parameter.

\section{Numerical Application}

We present in Table 1 the numerical data of the binary system follow by calculation of radiation pressure factors.

Table 1. Numerical Data for the Binary System.

\begin{tabular}{llllllll}
\hline Binary system & Masses $\left(M_{0}\right)$ & Eccent. (e) & Semi-major axis (a) & Lumino.. & Spec. Types & \\
\hline & $M_{1}$ & $M_{2}$ & & & $L_{1}$ & $L_{2}$ & \\
Xi Bootis & 0.9 & 0.66 & 0.5117 & 4.9044 & 0.49 & 0.061 & G8/k4 \\
Kruger 60 & 0.271 & 0.176 & 0.4100 & 2.3830 & 0.01 & 0.0034 & M3/M4 \\
\hline
\end{tabular}

Data source: NASA ADS

We now calculate the radiation pressure factor using the Setefan Baltzman's law [10] as $\mathrm{q}=1-(\Lambda \times \mathrm{L} / \mathrm{r} \rho \mathrm{M}), \mathrm{M}$ and $\mathrm{L}$ are the mass and luminosity of a star respectively; $r$ and $\rho$ are the radius and density of a moving body respectively, $q$ is the radiation pressure efficiency factor of a star, $\Lambda=\left(\frac{3}{16 \pi C G}\right)$ is a constant.In the C.G.S system $\Lambda=2.983810^{-5}$, We take $r=2 X 10^{-2}$ and $\rho=1.4 \mathrm{gcm}^{-3}[10]$ for some dust particle. The radiation factor obtained are $q_{1}=0.9988$ and $q_{2}=0.9998$. We substituted values of the parameters of the binary system into (12) and obtained triangular equilibrium points for the system, which are presented in Table 2, where in each case is $\mu=\frac{m 2}{m 1+m 2}$.

Table 2. Location of Triangular Equilibrium Points for the binary systems when $M_{b}=0.01, r_{c}=0.869, T=0.01$.

\begin{tabular}{|c|c|c|c|c|c|c|c|c|c|c|c|}
\hline Binary system & $\mu$ & $q_{1}$ & $q_{2}$ & $a$ & $e$ & $\sigma_{1}$ & $\sigma_{2}$ & $\sigma_{3}$ & $\sigma_{4}$ & $\xi$ & $\pm \eta$ \\
\hline \multirow[t]{3}{*}{ X1-Bootis } & 0.4231 & 0.9988 & 0.9998 & 0.7304 & 0.5117 & 0 & 0 & 0 & 0 & 0.0767004 & 0.590252 \\
\hline & & & & & & 0.004 & 0.02 & 0.003 & 0.011 & 0.0766489 & 0.577118 \\
\hline & & & & & & 0.008 & 0.006 & 0.01 & 0.03 & 0.0759019 & 0.576674 \\
\hline \multirow[t]{4}{*}{ Kruger 60} & 0.3937 & 0.9992 & 0.9996 & 0.5894 & 0.4100 & 0 & 0 & 0 & 0 & 0.31958 & 0.565314 \\
\hline & & & & & & 0.094 & 0.002 & 0.003 & 0.01 & 0.296101 & 0.544152 \\
\hline & & & & & & 0.06 & 0.004 & 0.005 & 0.02 & 0.288585 & 0.537201 \\
\hline & & & & & & 0.08 & 0.006 & 0.01 & 0.03 & 0.277940 & 0.527200 \\
\hline
\end{tabular}

Table 3. Effect of radiation Pressure on the Location of Triangular equilibrium points of xi-bootis $M_{b}=0.01, r_{c}=0.869, T=0.01$.

\begin{tabular}{|c|c|c|c|c|c|c|c|c|c|c|}
\hline$\mu$ & $\sigma_{1}$ & $\sigma_{2}$ & $\sigma_{3}$ & $\sigma_{4}$ & $\mathrm{e}$ & a & $q_{1}$ & $q_{2}$ & $\xi$ & $\pm \eta$ \\
\hline \multirow[t]{6}{*}{0.4231} & 0.004 & 0.002 & 0.08 & 0.02 & 0.7304 & 0.5117 & 0.99 & 0.95 & 0.0872081 & 0.4829534 \\
\hline & & & & & & & 0.85 & 0.80 & 0.0762394 & 0.4385567 \\
\hline & & & & & & & 0.80 & 0.75 & 0.0714364 & 0.4216989 \\
\hline & & & & & & & 0.75 & 0.70 & 0.0663268 & 0.403929 \\
\hline & & & & & & & 0.70 & 0.65 & 0.0608527 & 0.3851350 \\
\hline & & & & & & & 0.65 & 0.60 & 0.0549389 & 0.3651739 \\
\hline
\end{tabular}

Table 4. Effect of Radiation Pressure on critical mass value $\mu_{c}$ of xi booti $M_{b}=0.01, r_{c}=0.869, T=0.01$.

\begin{tabular}{|c|c|c|c|c|c|c|c|c|}
\hline A & $\mathrm{e}$ & $\sigma_{1}$ & $\sigma_{2}$ & $\sigma_{3}$ & $\sigma_{4}$ & $q_{1}$ & $q_{2}$ & $\mu_{c}$ \\
\hline \multirow[t]{3}{*}{0.7304} & 0.5117 & 0.001 & 0.02 & 0.0011 & 0.01 & 0.9968 & 0.9996 & 0.3160566188 \\
\hline & & & & & & 0.9978 & 0.9997 & 0.3160468096 \\
\hline & & & & & & 0.9988 & 0.9998 & 0.316037003 \\
\hline
\end{tabular}


Table 5. Effect of triaxiality on Stability range of critical mass value $u_{c}$ of Kruger $60 M_{b}=0.01, r_{c}=0.869, T=0.01$.

\begin{tabular}{|c|c|c|c|c|c|c|c|c|}
\hline A & $\mathrm{e}$ & $\sigma_{1}$ & $\sigma_{2}$ & $\sigma_{3}$ & $\sigma_{4}$ & $q_{1}$ & $q_{2}$ & $\mu_{c}$ \\
\hline \multirow[t]{5}{*}{0.96} & 0.03 & 0.001 & 0.004 & 0.002 & 0.01 & 0.99 & 0.95 & 0.0363732 \\
\hline & & 0.002 & 0.0041 & 0.0022 & 0.011 & & & 0.0196754 \\
\hline & & 0.00201 & 0.004101 & 0.00221 & 0.0111 & & & 0.0195519 \\
\hline & & 0.00202 & 0.004102 & 0.00222 & 0.0112 & & & 0.0194285 \\
\hline & & 0.00203 & 0.004103 & 0.00223 & 0.0113 & & & 0.0193050 \\
\hline
\end{tabular}

Table 6. The effect of mass ratio and the disc on the characteristic roots of the binary system (xi-bootis) when $\sigma_{1}=0.001, \sigma_{2}=0.01, \sigma_{3}=0.002$ $\sigma_{4}=0.02, q_{1}=0.9988$ and $q_{2}=0.9998 r_{c}=0.968 T=0.1$.

\begin{tabular}{llll}
\hline Mass ratio $\mu$ & $\lambda_{i}\left(M_{b}=0\right)$ & $\lambda_{i}\left(M_{b}=0.05\right)$ & $\lambda_{i}\left(M_{b}=0.1\right)$ \\
\hline 0.0005 & $\pm 1.49626 \pm 0.311611$ & $\pm 0.503409 \mathrm{i} \pm 1.56672 \mathrm{i}$ & $\pm 1.65723 \mathrm{i} \pm 2.237231 \mathrm{i}$ \\
0.005 & $\pm 1.48601 \pm 0.359146$ & $\pm 0.539042 \mathrm{i} \pm 1.55439 \mathrm{i}$ & $\pm 1.67029 \mathrm{i} \pm 2.22728 \mathrm{i}$ \\
0.017 & $\pm 1.45714 \pm 0.466375$ & $\pm 0.628694 \mathrm{i} \pm 1.51917 \mathrm{i}$ & $\pm 1.70698 \mathrm{i} \pm 2.19847 \mathrm{i}$ \\
0.027 & $\pm 1.43099 \pm 0.544069$ & $\pm 0.700349 \mathrm{i} \pm 1.4865 \mathrm{i}$ & $\pm 1.74041 \mathrm{i} \pm 2.17143 \mathrm{i}$ \\
0.034 & $\pm 1.41124 \pm 0.59165$ & $\pm 0.700332 \mathrm{i} \pm 1.4612 \mathrm{i}$ & $\pm 1.76607 \mathrm{i} \pm 2.150121 \mathrm{i}$ \\
0.0384 & $\pm 1.39808 \pm 0.626489$ & $\pm 0.782166 \mathrm{i} \pm 1.44396 \mathrm{i}$ & $\pm 1.78355 \mathrm{i} \pm 2.13534 \mathrm{i}$ \\
0.03851 & $\pm 1.39774 \pm 0.627267$ & $\pm 0.782969 \mathrm{i} \pm 1.44351 \mathrm{i}$ & $\pm 1.784 \mathrm{i} \pm 2.13495 \mathrm{i}$ \\
0.03852 & $\pm 1.39771 \pm 0.627338$ & $\pm 0.783042 \mathrm{i} \pm 1.44347 \mathrm{i}$ & $\pm 1.78405 \mathrm{i} \pm 2.13492 \mathrm{i}$ \\
0.03853 & $\pm 1.39768 \pm 0.627408$ & $\pm 0.783115 \mathrm{i} \pm 1.44343 \mathrm{i}$ & $\pm 1.78409 \mathrm{i} \pm 2.13488 \mathrm{i}$ \\
0.07 & $\pm 1.27242 \pm 0.858737$ & $\pm 1.0962 \mathrm{i} \pm 1.21893 \mathrm{i}$ & $\pm 0.0996526 \pm 1.96866 \mathrm{i}$ \\
0.09 & $\pm 1.09202 \pm 0.10634 \mathrm{i}$ & $\pm 0.229649 \pm 1.18047 \mathrm{i}$ & $\pm 0.185036 \pm 1.97408 \mathrm{i}$ \\
0.11942 & $\pm 1.12441 \pm 0.280589 \mathrm{i}$ & $\pm 0.352046 \pm 1.20902 \mathrm{i}$ & $\pm 0.260712 \pm 1.9815 \mathrm{i}$ \\
\hline
\end{tabular}

Table 7. The characteristic root under the combine effect of triaxiality,radiation and the pontetial from the belt for the binary system xi-bootis when $\sigma_{1}=0.04, \sigma_{2}=0.03, \sigma_{3}=0.01, \sigma_{4}=0.02, q_{1}=0.9988$ and $, q_{2}=0.9998, T=0.1, r_{c}=0.869$.

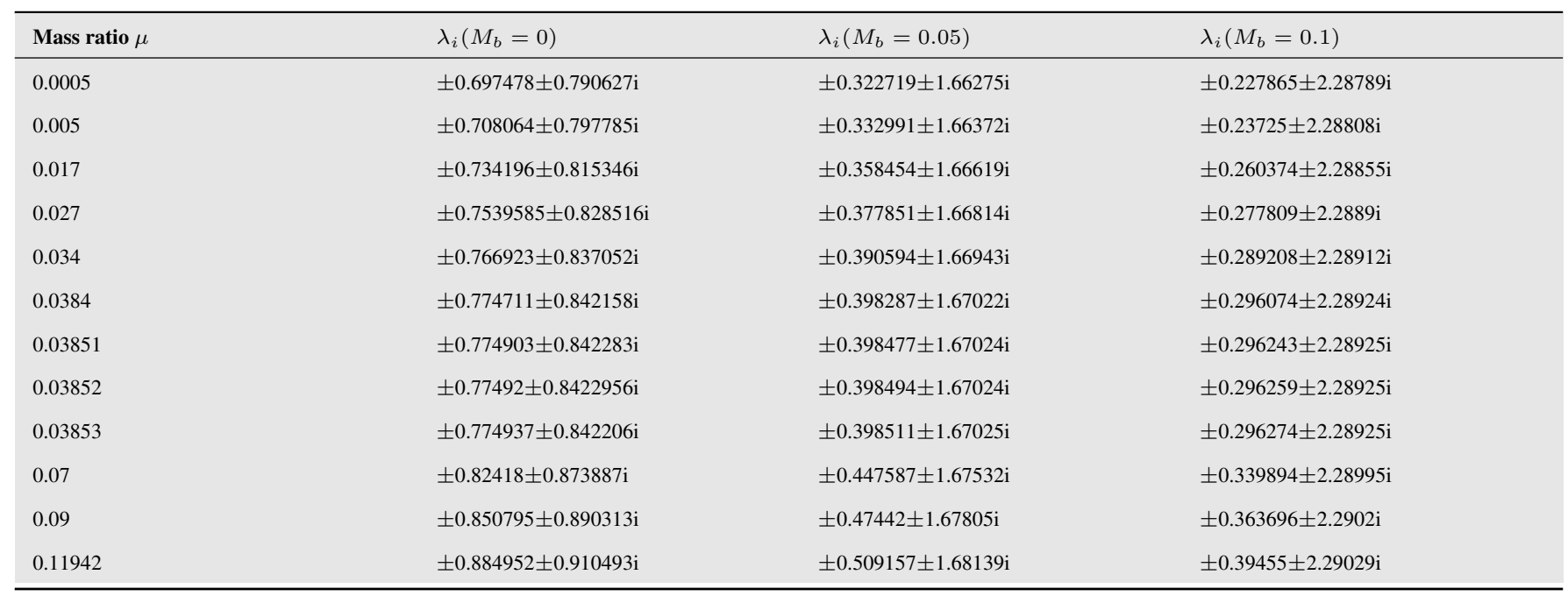




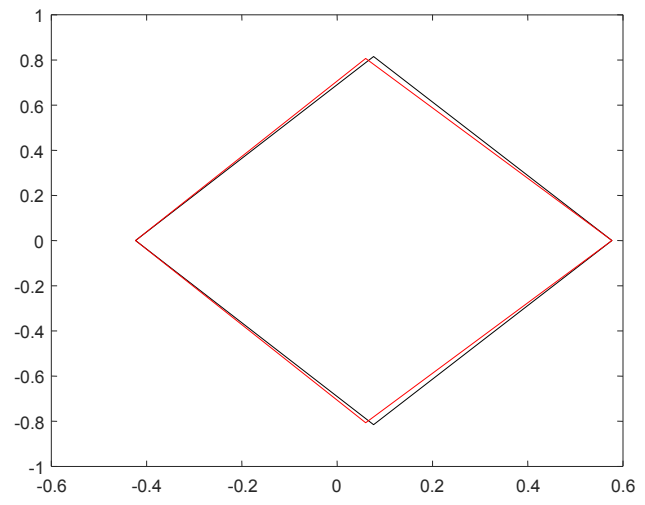

Figure 1. The effect of triaxiality on the $L_{4,5}$ of xi booti.

Figure 1. Showing effect of trixiality on $L_{4,5}$ of xi-bootis when $\sigma_{i}=(i=1,4)=0, q_{1}=0.9988, q_{2}=0.9998 . a=$ $0.7304, e=0.5117$ and when $\sigma_{1}=0.004, \sigma_{2}=0.002, \sigma_{3}=$ $0.003, \sigma_{4}=0.003, M_{b}=0.1, T=0.1, r_{c}=0.869$

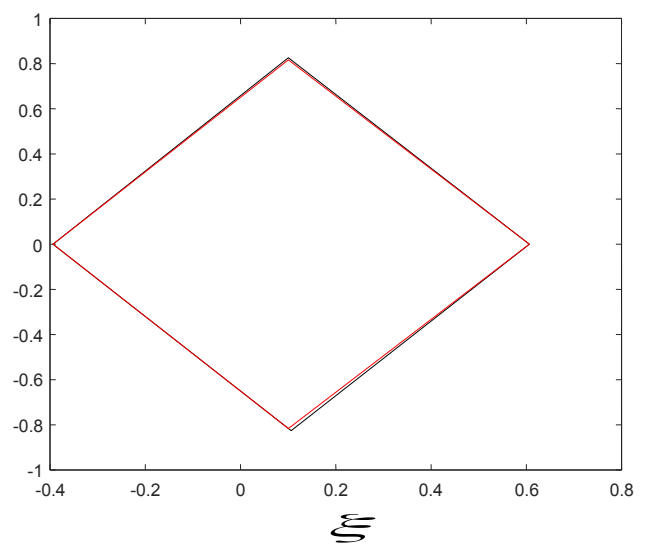

Figure 2. Effect of triaxiality on triangular point for kruger.60.

Figure 2. Showing the effect oftriaxiality on triangular equilibrium points for Kruger $60 \sigma_{i}=(i=1,4)=0, q_{1}=$ $0.9992, q_{2}=0.9996 . a=0.5894, e=0.4100$ and when $\sigma_{1}=0.004, \sigma_{2}=0.002, \sigma_{3}=0.003, \sigma_{4}=0.01, M_{b}=$ $0.01, T=0.01, r_{c}=0.869$

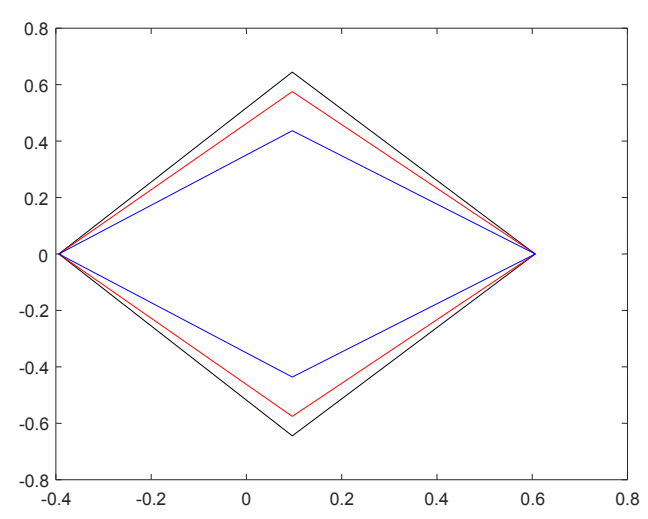

Figure 3. Effect of semi major axis on $L_{4,5}$ of xi-booti.
Figure 3. Showing Effect of semi major axis on $L_{4,5}$ of xi-bootis when $q_{1}=0.9988, q_{2}=0.9998 \mu=0.4231, e=$ 0.5117 and when $\sigma_{1}=0.04, \sigma_{2}=0.02, \sigma_{3}=0.003, \sigma_{4}=$ $0.005, M_{b}=0.01, T=0.01, r_{c}=0.869$ for $a=$ $0.37,0.68,0.97$

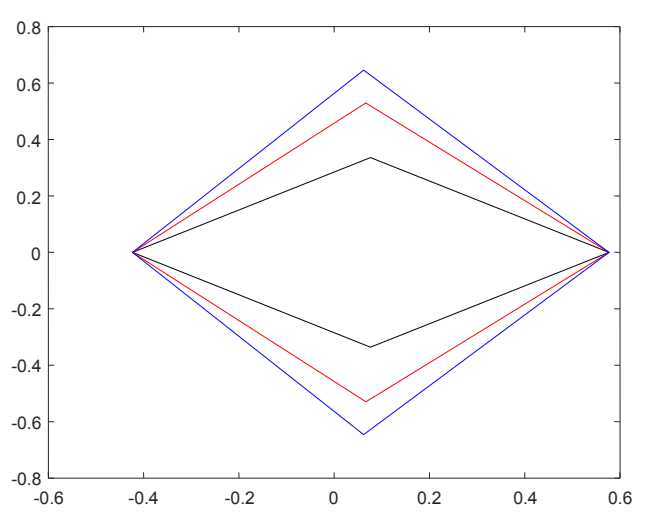

Figure 4. Effect of eccentricity on $L_{4,5}$ of kruger 60.

Figure 4. showing effect of eccentricity on $L_{4,5}$ of kruger 60 when $q_{1}=0.9992, q_{2}=0.9998, \mu=0.3937, a=$ $0.5894, \sigma_{1}=0.04, \sigma_{2}=0.02, \sigma_{3}=0.003, \sigma_{4}=$ $0.005, M_{b}=0.01, T=0.01, r_{c}=0.869$ fore $=0.3,0.6,0.9$

\section{Discussion and Conclusion}

The motion of an infinitesimal mass around $L_{4,5}$ of the triangular equilibrium points have been investigated in the framework of ER3BP taking both the primaries as radiating and triaxial with gravitational potential from the belt .We have used (12) to establish triangular equilibrium points for the binary system (xi-bootis and kruger 60), these are presented in Table 2. It can be seen there that the positions of equilibrum points shift toward the $\xi$ axis as triaxiality effect increases. The radiation pressures used in the Table 3 were taken from [19]. It is observed in Table 3 that decreasing the radiation pressure from 0.99 and 0.95 to 0.65 and 0.60 shift the triangular equilibrium point towards the $\xi$-axis[19]. Similar effects occurs in Table 4 where increases in radiation pressure lead to a decrease of the critical mass value $\mu_{c}$ and consequently the stability region. This destabilizing tendency is also exhibited by the triaxial nature of the primaries as shown in Table 5 where the effect of triaxiality reduces the range of the critical mass ratio.

We compute the characteristic roots of xi- Bootis using (21) and present the result in Table 6 and 7 for some arbitrary values of mass rato,triaxiality,radiation pressure and the disc.We consider the range $0 \leq \mu \leq 0.11942$ for the mass ratio this is to enable us observe the behaviour of the system parameters when $0<\mu \leq 0.03852$, the stability range of restricted three body problem. We found that in the absence of the disc $M_{b}=0$ the roots are real numbers except few that are complex roots but in the presence of the disc $M_{b}=0.05$ and $M_{b}=0.1$ the roots becomes pure imaginary numbers.This 
confirms the stability effect of the circumbinary disc.However using the same range of mass ratio the combine effects of triaxiality,radiation pressure and disc yielded complex roots despite the presence of the disc leading to instability of the triangular equilibrium points under investigation.

A graphical representation of the effect of triaxiality on $L_{4,5}$ of xi-bootis and kruger 60 is shown in Figure 1 and Figure 2 using MATLAB 2016.The coordinates of $L_{4,5}$ shift towards the $\xi$ axis, when triaxiality was introduced from its previous position when triaxiality was absent showing the destabilizing effect of triaxiality on the positions of $L_{4,5}$.

We show the effect of semi-major axis and eccentricity on the location of triangular equilibrium points of xi-bootis and kruger 60 respectively in Figure 3 and Figure 4. It can be seen clearly that their positions are shifting away from the $\xi$ axis as their effect increases. This pertubing effect was also observed by [15] in their paper. In [18] the triangular point was unstable when the value of other parameters were increased but becomes stable on introducing the pontetial from the belt confirming its stabilizing potential.

The coordinates of our triangular equilibrium points coincides with triangular equilibrium points of [10] when both primaries are oblate spheroids i.e $\sigma_{1}=\sigma_{2}$ and $\sigma_{3}=\sigma_{4}$ and $M_{b}=0$. It also agrees with triangular equilibrium points of [8] if $q_{i}=1-p i$, (i=1,2) $a=1, e=0, M_{b}=0$. If we put $q_{i}=1-p i$ (i=1,2), $a=1, e=0$ and when $\sigma_{1}=\sigma_{2}$ and $\sigma_{3}=\sigma_{4}$ we get the same results as triangular equilibrium points of [13]. when both primaries are oblate spheroids and radiating with gravitational pontetial from the belt with circular orbits.

Equation (23) gives the value for the critical mass ratio $\mu_{c}$ and is a function of the combined effects of radiation forces,triaxiality and gravitational potential from the belt. The value of critical mass ratio $\mu_{c}$ determines the range of the stability of the system. When both primaries are oblate our critical mass ratio $\mu_{c}$ tallies with critical mass ratio $\mu_{c}$ of [10]. When $\sigma_{1}=\sigma_{2}$ and $\sigma_{3}=\sigma_{4}$ and $M_{b}=0$. Similarly, if we put $\beta_{1}=\beta_{2}=0, \sigma_{1}=\sigma_{2}$ and $\sigma_{3}=\sigma_{4}, M_{b}=0$ in equation (23) we get the same results as critical mass $\mu_{c}$ of [23] up to zonal harmonics $J_{2}$ when both primaries are oblate with elliptic orbit.

\section{References}

[1] Nutan, S., Narayan, A. and Ishwar, B (2015) 'Trajectoies of the infinitesimal mass around the triangular equilibrium points in ERT3BP under oblate and radiating primaries for the binary systems', International Journal of Advanced Astronomy, Vol. 3,No. 2, pp 97-106

[2] Szebehely, V. G. (1967) Theory of Orbits, Academic Press, New York, London.

[3] Zimovschikov, A. S. and Tkhai, V. N (2004) 'Instability of libration points and resonance phenomena in the photogravitational Elliptic restricted three- body problem', Solar Systems Research, Vol. 38, p155.
[4] Pandey, K. K, Narayan, A. and Shivastava, S. K (2019) 'Effect of Triaxiality and radiation pressure on Existence of Resonance Stability of Triangular Equilibrium Points in Elliptical Restricted Three-Body Problem', Journal of Informatics and Mathematical Sciences Vol. 11, pp 3-4.

[5] Singh, J. and Haruna, S. (2020) ¡®Motion around triangular points in the Restricted Three-Body problem with radiating heterogeneous primaries surrounded by a belt ${ }^{-}$, Science Reports, Vol 10,18861.Doi/10.1038/S41598-020-7517 pp 4-7

[6] Narayan, A. and Nutan, S.(2014)'Stability of triangular Lagragian points in the elliptic restricted three-body problem under radiating binary system', Astrophysics and Space Science Vol. 353, No. 2, pp 457-464

[7] Narayan,A. and Ramesh, C. (2011) 'Stability of Triangular Equilibrium points in elliptical restricted three-body problem under effects of photogravitational and oblateness of primaries', International journal of pure and applied Mathematics, Vol. 70 pp 735.

[8] Selim, H. H.,Guirao,J. L. G. and Abouelmagd, E. I. (2019) $; \circledR$ Libration Points in the Restricted Three BodyProblem:Euler angles,existence and stability ${ }^{-}$, Discrete and Continous Dynamical systems 12 ( 4 and 5) pp 703710 .

[9] Singh, J. and Umar, A. (2012a) 'Motion in the photogravitational elliptic restricted three- body problem under oblate primaries',The Astronomical Journal, Vol. 143 pp 109-131.

[10] Singh, J. and Umar, A. (2012b) 'On the stability of triangular points in the elliptic restricted threebody problem under radiating and oblate primaries', Astrophysics and Space Science, Vol. 341, pp 349-358.

[11] Arif, M and Sagar, R. K (2018)i ${ }^{-}$Study of the Restricted Three-Body Problem when the Primary is a uniform circular Disk ${ }^{-}$Application and Applied Mathematics Vol. 13, 1 pp 160-172.

[12] Khanna, M. and Bhatnagar, K. B. (1999) 'Existence and Stability of libration points in the stricted three-body problem when the smaller primary is a triaxial rigid body and the Bigger one an oblate spheroids', India journal of pure and applied Mathematics, Vol. 30 No. 7 pp 721-733.

[13] Singh, J. and Taura, J. J. (2014b) 'Combine effect of oblateness,radiation and a cluster of material points on the stability of triangular libration points in the restricted three-body problem', Journal of Astrophysics and Astronomy, Vol. 351, No. 2. Doi 10.1007/s 10509014-1860-8

[14] Narayan, A., Pandey, K. K. and Shrivastava, S. K. (2015)` Effects of radiation and triaxiality of triangular points in elliptical restricted three-body problem', International Journal of Advanced Astronomy, Vol. 3, No. 2, pp 97-106 
[15] Umar, A. and Hussain, A. A (2016) 'Motion in the elliptic restricted three- body problem with an oblate primary and triaxial stellar companion', Astrophysics and Space Science, Vol. 361, No. 344

[16] Singh, J. and Taura, J. J. (2014c) 'Effects of triaxiality, oblateness and gravitational pontetial from a belt on the linear stability of $\mathrm{L}_{4,5}$ in the restricted three-body problem', Journal of Astrophysics and Astronomy, Vol. 35 , No. 4 pp 729-743

[17] Singh, J. and Taura, J. J. (2014a) 'Stability of triangular equilibrium points in the photogravitational restricted three-body problem with oblateness and pontetial from a belt',Journal of Astrophysics and Astronomy, Vol. 35 pp 107-109.

[18] Singh, J. and Amuda, T. O. (2019)` Stability Analysis of Triangular equilibrium points inrestricted three-body problem under effect of circumbinary disc,radiation and drag forces',Astrophysics and astronomy, Vol. 40, No. 5 http://doi.org/10.1007/sDoi10.1007/s 12036-019-9537-6
[19] Singh, J. (2013) 'The Equilibrium points in perturbed restricted three-body problem with triaxial and luminous primaries', Astrophysics and Space Science, Vol. 346 pp $41-50$

[20] Kushvah, B. S. (2008)'Linear Stability of equilibrium points in the generalized photogravitational chermnykh's problem', Astrophysics and Space Science, Vol. 318, pp 41-50.

[21] Miyamoto, M. and Nagai, P. (1975) 'Three Dimensional Models for the distribution of mass galaxies', ,Astronomical Society of Japan, Vol. 27, pp 533-543.

[22] Jiang, I. G. and Yeh, L. C. (2003)Birfurcation for dynamical systems of planet-belt interaction, International Journal of Birfucation and Chaos, 13 (3) 617-630.

[23] Singh, J. and Tokyaa, R. K. (2016) 'Stability of Triangular points in the ellipticrestricted three-body problem with oblateness up to zonal harmonics J4 of both primaries', The European physical Journal plus, Vol. 131, pp 1-11. 